\title{
EXTRAORDINARY LUMINOUS SOFT X-RAY TRANSIENT MAXI J0158-744 AS AN IGNITION OF A NOVA ON A VERY MASSIVE O-Ne WHITE DWARF
}

\author{
M. MoriI ${ }^{1}$, H. Tomida ${ }^{2}$, M. Kimura ${ }^{2}$, F. Suwa ${ }^{3}$, H. Negoro ${ }^{3}$, M. Serino ${ }^{1}$, J. A. Kennea ${ }^{4}$, K. L. Page ${ }^{5}$, P. A. Curran $^{6}$, \\ F. M. Walter ${ }^{7}$, N. P. M. Kuin ${ }^{8}$, T. Pritchard ${ }^{4}$, S. Nakahira ${ }^{2}$, K. Hiroi ${ }^{9}$, R. Usui ${ }^{10}$, N. Kawai ${ }^{10}$, J. P. Osborne ${ }^{5}$, T. Mihara ${ }^{1}$, \\ M. Sugizaki ${ }^{1}$, N. Gehrels ${ }^{11}$, M. Kohama ${ }^{2}$, T. Kotani ${ }^{12}$, M. Matsuoka ${ }^{1}$, M. Nakajima ${ }^{13}$, P. W. A. Roming ${ }^{14}$, T. SaKamoto ${ }^{15}$,

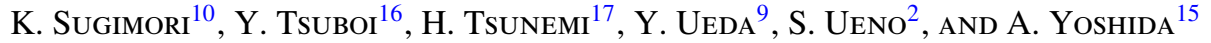 \\ ${ }^{1}$ MAXI Team, Institute of Physical and Chemical Research (RIKEN), 2-1 Hirosawa, Wako, Saitama 351-0198, Japan \\ ${ }^{2}$ ISS Science Project Office, Institute of Space and Astronautical Science, Japan Aerospace Exploration Agency, 2-1-1 Sengen, Tsukuba, Ibaraki 305-8505, Japan \\ ${ }^{3}$ Department of Physics, Nihon University, 1-8-14 Surugadai, Chiyoda, Tokyo 101-8308, Japan \\ ${ }^{4}$ Department of Astronomy and Astrophysics, The Pennsylvania State University, 525 Davey Laboratory, University Park, PA 16802, USA \\ ${ }_{5}^{5}$ Department of Physics and Astronomy, University of Leicester, University Road, Leicester LE1 7RH, UK \\ ${ }^{6}$ International Centre for Radio Astronomy Research, Curtin University, GPO Box U1987, Perth, WA 6845, Australia \\ ${ }^{7}$ Department of Physics and Astronomy, Stony Brook University, Stony Brook, NY 11794-3800, USA \\ ${ }_{8}^{8}$ Mullard Space Science Laboratory, University College London, Holmbury St Mary, Dorking, Surrey RH5 6NT, UK \\ ${ }^{9}$ Department of Astronomy, Kyoto University, Oiwake-cho, Sakyo-ku, Kyoto 606-8502, Japan \\ ${ }^{10}$ Department of Physics, Tokyo Institute of Technology, Ookayama 2-12-1, Meguro-ku, Tokyo 152-8551, Japan \\ ${ }^{11}$ NASA Goddard Space Flight Center, Greenbelt, MD 20771, USA \\ ${ }^{12}$ Waseda University, Organization for University Research Initiatives, 17 Kikuicho, Shinjuku, Tokyo 162-0044, Japan \\ ${ }^{13}$ School of Dentistry at Matsudo, Nihon University, 2-870-1 Sakaecho-nishi, Matsudo, Chiba 271-8587, Japan \\ ${ }^{14}$ Southwest Research Institute, Space Science and Engineering Division, PO Drawer 28510, San Antonio, TX 78228-0510, USA \\ ${ }^{15}$ Department of Physics and Mathematics, Aoyama Gakuin University, 5-10-1 Fuchinobe, Chuo-ku, Sagamihara, Kanagawa 252-5258, Japan \\ ${ }^{16}$ Department of Physics, Faculty of Science and Engineering, Chuo University, 1-13-27 Kasuga, Bunkyo-ku, Tokyo 112-8551, Japan \\ ${ }^{17}$ Department of Earth and Space Science, Osaka University, 1-1 Machikaneyama, Toyonaka, Osaka 560-0043, Japan \\ Received 2013 April 24; accepted 2013 October 3; published 2013 December 2
}

\begin{abstract}
We present the observation of an extraordinary luminous soft X-ray transient, MAXI J0158-744, by the Monitor of All-sky X-ray Image (MAXI) on 2011 November 11. This transient is characterized by a soft X-ray spectrum, a short duration $\left(1.3 \times 10^{3} \mathrm{~s}<\Delta T_{d}<1.10 \times 10^{4} \mathrm{~s}\right)$, a rapid rise $\left(<5.5 \times 10^{3} \mathrm{~s}\right)$, and a huge peak luminosity of $2 \times 10^{40} \mathrm{erg} \mathrm{s}^{-1}$ in $0.7-7.0 \mathrm{keV}$ band. With Swift observations and optical spectroscopy from the Small and Moderate Aperture Research Telescope System, we confirmed that the transient is a nova explosion, on a white dwarf in a binary with a Be star, located near the Small Magellanic Cloud. An early turn-on of the super-soft X-ray source (SSS) phase ( $<0.44$ days), the short SSS phase duration of about one month, and a $0.92 \mathrm{keV}$ neon emission line found in the third MAXI scan, $1296 \mathrm{~s}$ after the first detection, suggest that the explosion involves a small amount of ejecta and is produced on an unusually massive O-Ne white dwarf close to, or possibly over, the Chandrasekhar limit. We propose that the huge luminosity detected with MAXI was due to the fireball phase, a direct manifestation of the ignition of the thermonuclear runaway process in a nova explosion.
\end{abstract}

Key words: Magellanic Clouds - novae, cataclysmic variables - stars: individual (MAXI J0158-744) - white dwarfs - X-rays: bursts - X-rays: individual (MAXI J0158-744)

Online-only material: color figure

\section{INTRODUCTION}

Classical or recurrent novae are typically characterized by a rapid optical increase of 6 magnitudes or more, followed by a decline to quiescence over the next 3-300 days (Warner 1995). They originate from an accreting binary system consisting of a white dwarf (WD) and a mass-losing, late-type companion star. Novae are triggered by thermonuclear runaways (TNRs) lasting $\sim 100 \mathrm{~s}$ at the bottom of the accreted mass layer on the WD surface (Warner 1995; Starrfield et al. 2008). The TNR blows off the outer layer of the accumulated mass and causes an optically thick wind expanding up to $\sim 100 R_{\odot}$. It produces bright blackbody emission $\left(\sim 10^{38} \mathrm{erg} \mathrm{s}^{-1}\right.$, comparable to the Eddington luminosity of a $1 M_{\odot}$ object) at optical bands. This optical nova phase lasts for $\sim 3-300$ days (Warner 1995). At the same time, a blast wave, caused by a nova explosion in a dense circumstellar medium, sometimes produces shock- induced, optically thin hard X-ray emission lasting $\sim 10$ days, as observed in RS Ophiuchi (Sokoloski et al. 2006) and V407 Cyg (Nelson et al. 2012), for example. After the wind stops, the photosphere shrinks down to the WD surface $\left(\sim 10^{3}-10^{4} \mathrm{~km}\right)$, and the blackbody temperature increases to $\sim 100 \mathrm{eV}$, meaning the emission is in the soft X-ray energy range. This transient phase with soft X-ray emission is called the super-soft source phase (SSS phase) and it lasts about $\sim 100-1000$ days (Schwarz et al. 2011; Hachisu \& Kato 2006). When the nuclear burning stops, the SSS phase ends. Novae are classified into speed classes according to the decay time scale of their optical light curves (Warner 2008). Faster novae show earlier turn-ons and shorter durations of the SSS phase. For example, the fastest nova, U Sco, showed a turn-on of the SSS phase at 10 days and had a duration of about 25 days (Schwarz et al. 2011). In general, the evolution of classical/recurrent novae has been established, except for the early phase. At the time of the TNR, the early and short emission 

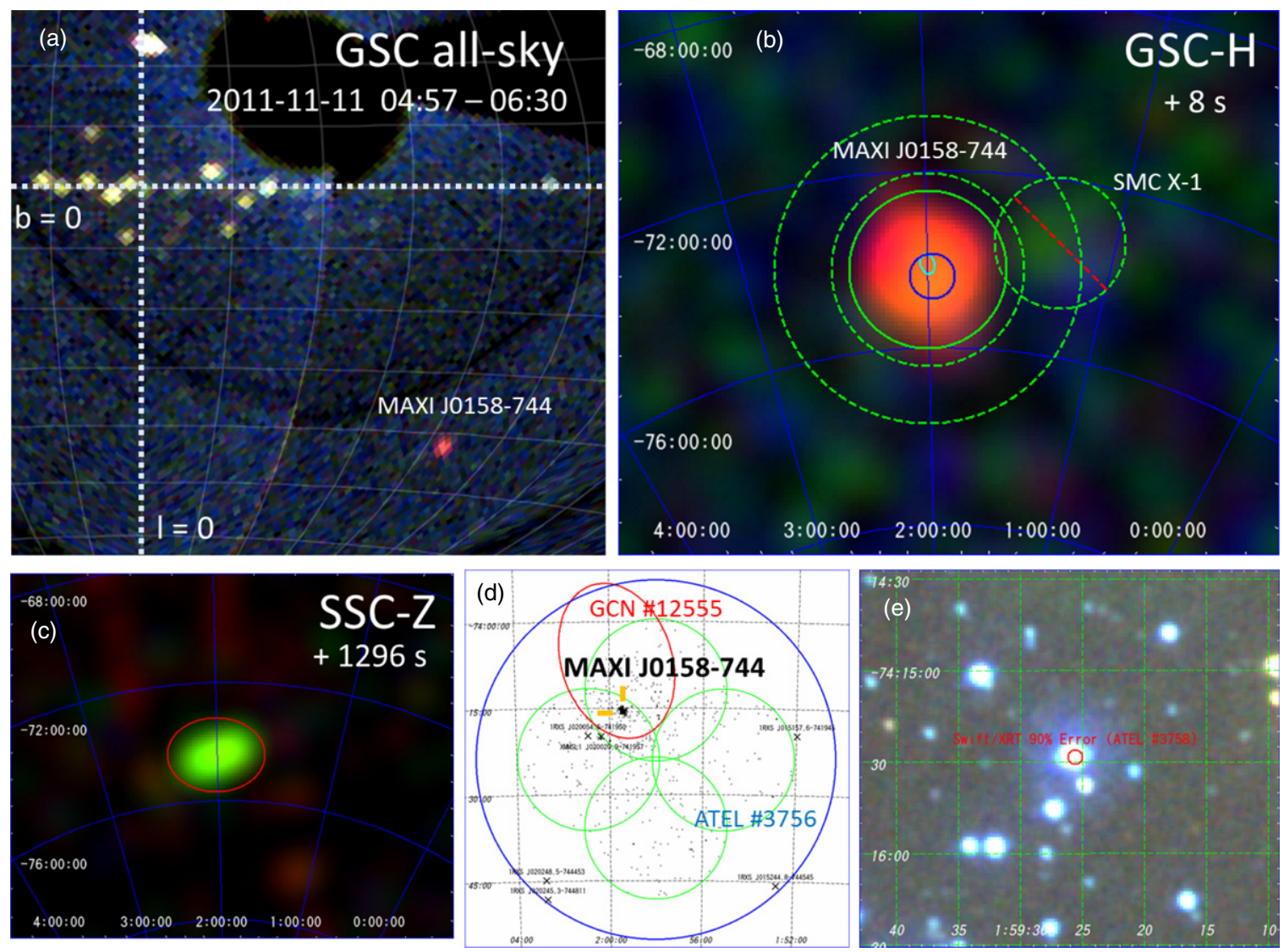

Figure 1. Images of MAXI J0158-744 taken by MAXI and Swift. (a) A part of the GSC all-sky image shown in Hammer-Aitoff projection of Galactic coordinates. Red, green, and blue color maps represent the intensities in 2-4, 4-10, and 10-20 keV bands, respectively. Grid lines are drawn every $1 \mathrm{hr}$ and $10^{\circ}$ in longitude and latitude, respectively. (b) Scan image of GSC-H around $T_{\text {trig. }}$. The source and background regions for the spectral analysis are shown in a green solid circle and dashed annulus, respectively. The excluded region centered at SMC X-1 is shown by a dashed circle. The colors represent the same energy bands as in (a). The error regions reported in Kimura et al. (2011) and Morii et al. (2011b) are shown by a blue circle and cyan ellipse, respectively. Here, these error regions are made by adding the $90 \%$ confidence statistical error and the systematic uncertainty ( $0.1,90 \%$ containment radius). (c) Scan image of SSC-Z around $T_{\text {trig }}+1296 \mathrm{~s}$. The source regions are shown by the red ellipses. Red, green, and blue color maps represent the intensities in 0.7-1, 1-4, and 4-10 keV bands, respectively. (d) Swift XRT mosaic image around MAXI J0158-744 obtained by the tiling observation data taken to search the X-ray counterpart (Kennea et al. 2011a). Green circles show FoVs of four Swift XRT pointings. The X-ray sources cataloged in ROSAT All-sky Survey and XMM-Newton Slew Survey are marked with X symbols. The same error regions as (b) are shown by blue circle and red ellipse. (e) Swift UVOT image made by combining the data with $v, b$, and $u$ filters, colored in red, green, and blue, respectively. The $90 \%$ confidence error circle obtained by Swift XRT is shown by a red circle.

(A color version of this figure is available in the online journal.)

(a few hours) is predicted to appear in the ultraviolet (UV) to soft X-ray range, called "fireball phase" (Starrfield et al. 1998, 2008; Krautter 2008a, 2008b). However, no such signal has been observed because of the difficulty in detecting the abrupt short phenomenon appearing in these energy range.

Monitor of All-sky X-ray Image (MAXI; Matsuoka et al. 2009) is an all-sky X-ray monitor, which is operated on the Japanese Experiment Module, the Exposed Facility on the International Space Station (ISS). MAXI carries two types of X-ray cameras: Gas Slit Camera (GSC; Mihara et al. 2011; Sugizaki et al. 2011) and Solid-state Slit Camera (SSC; Tsunemi et al. 2010; Tomida et al. 2011). GSC and SSC have wide fields of view (FoVs) of $1.5 \times 160^{\circ}$ and $1.5 \times 90^{\circ}$, respectively. They scan almost all of the sky every $\sim 92$ minutes using the attitude rotation coupled with the ISS orbital motion (see Figure 1 in Sugizaki et al. 2011). GSC covers the $2-30 \mathrm{keV}$ band using gas proportional counters, while SSC covers the $0.5-12 \mathrm{keV}$ band with the X-ray charge-coupled devices (CCDs). MAXI started its operation in orbit in August of 2009.

The MAXI transient alert system (Negoro et al. 2010) was triggered on 2011 November 11 at 05:05:59 UT ( $=T_{\text {trig }}$ ) by a new bright soft X-ray source near the Small Magellanic
Cloud (SMC; Figure 1(a)). ${ }^{18}$ We analyzed the data and reported the source position through an Astronomer's Telegram (ATEL; Kimura et al. 2011) and the GRB Coordinates Network (GCN; Morii et al. 2011b).

At 0.44 days after $T_{\text {trig }}$, Swift X-ray Telescope (XRT; Gehrels et al. 2004; Burrows et al. 2005) began follow-up observations (Kennea et al. 2011b) with a tiling mode to cover the MAXI error circle (Figure 1(d); Kimura et al. 2011). An uncataloged X-ray source was found within the MAXI GSC error ellipse (Figure 1(d); Kennea et al. 2011a; Morii et al. 2011b). Within the Swift XRT error circle, a single optical source is cataloged in USNO-A2.0, which was also reported as a source with a near-infrared excess (ID 115 in Nishiyama et al. 2007). The position is consistent with that of an optical counterpart observed by Swift Ultraviolet/Optical Telescope (UVOT; Roming et al. 2005), $\alpha=01^{\mathrm{h}} 59^{\mathrm{m}} 25^{\mathrm{s}} .83, \delta=-74^{\circ} 15^{\prime} 27^{\prime \prime} .9$, with an estimated uncertainty of $0.5 \operatorname{arcsec}(90 \%$ confidence; Figure 1(e); Kennea et al. 2011a).

\footnotetext{
18 GCN Notice: http://gcn.gsfc.nasa.gov/other/39.maxi; MAXI alert mailing list [New-transient:39]: http://maxi.riken.jp/pipermail/new-transient/ 2011-November/000038.html
} 
Table 1

Summary of MAXI Observation

\begin{tabular}{lccccc}
\hline \hline Scan-ID & Scan Time(Start-End)(UT) & $\Delta t(\mathrm{~s})^{\mathrm{a}}$ & $t(\mathrm{~s})^{\mathrm{b}}$ & Detector & Flux $^{\mathrm{c}}$ \\
\hline $\mathrm{M}-1^{\mathrm{d}}$ & $2011-11-1103: 33: 22-03: 34: 17^{*}$ & 55 & -5530 & GSC-H & $<2.75 \times 10^{-9} \quad \mathrm{e}, \mathrm{f}$ \\
$\mathrm{M}+0$ & $2011-11-1105: 05: 39^{\dagger}-05: 06: 34$ & 55 & +8 & GSC-H & $1.81_{-0.33}^{+0.14} \times 10^{-8} \mathrm{~g}$ \\
$\mathrm{M}+1$ & $2011-11-1105: 09: 13-05: 10: 04$ & 51 & +220 & SSC-H & $4.16_{-1.20}^{+0.47} \times 10^{-8} \mathrm{~g}$ \\
$\mathrm{M}+2$ & $2011-11-1105: 27: 09-05: 28: 00^{\ddagger}$ & 51 & +1296 & SSC-Z & $1.57_{-0.18}^{+0.17} \times 10^{-8} \mathrm{~h}$ \\
$\mathrm{M}+3$ & $2011-11-1106: 37: 56^{\S}-06: 38: 51$ & 55 & +5545 & GSC-H & $<9.60 \times 10^{-10} \mathrm{e}, \mathrm{f}$ \\
\hline
\end{tabular}

Notes.

${ }^{a}$ Total length of the MAXI scan.

b Time center of the MAXI scan referred to the trigger time $T_{\text {trig }}(=2011-11-11$ 05:05:59 UT).

${ }^{\mathrm{c}}$ Unabsorbed flux is in units of $\mathrm{erg} \mathrm{s}^{-1} \mathrm{~cm}^{-2}$ in an energy range of $0.7-7.0 \mathrm{keV}$.

d The last scan before $T_{\text {trig. }}$.

${ }^{\mathrm{e}}$ A blackbody model with a temperature of $0.37 \mathrm{keV}$ is assumed (Section 3.3).

${ }^{\mathrm{f}}$ Upper limit is $90 \%$ confidence.

$\mathrm{g}$ The best-fit blackbody model shown in Table 2 .

h The Blackbody + Mekal model with parameters shown in Section 3.2.2 is assumed.

* We set this time $t_{-1} \cdot{ }^{\dagger} t_{1} \cdot{ }^{\dagger} t_{2} \cdot{ }^{\S} t_{3}$.

The Swift XRT spectra obtained after $T_{\text {trig }}+0.44$ days were reported to be similar to the SSS phase of novae ( $\mathrm{Li}$ et al. 2012). Further follow-up observations by Swift and groundbased optical observations confirmed that this source is a binary system consisting of a WD and a Be star at the distance of the SMC ( $d=60 \mathrm{kpc}$; Hilditch et al. 2005; Li et al. 2012). Li et al. (2012) analyzed the spectrum of the GSC scan at +8 s, using the on-demand data products provided by the MAXI team, and reported that the luminosity was $\sim 1.6 \times 10^{39} \mathrm{erg} \mathrm{s}^{-1}$ in the 2-4 keV band; this is one order of magnitude brighter than the Eddington luminosity of a solar mass object. To explain the huge outburst luminosity, Li et al. (2012) proposed a model of the interaction of the ejected nova shell with the Be star wind in which the WD is embedded.

Here, we present observations of MAXI J0158-744 by MAXI, Swift and the Small and Moderate Aperture Research Telescope System (SMARTS) in Section 2. The analysis and results of the MAXI GSC and SSC data are described in Sections 3.1 and 3.2, with the detailed spectral analysis for the third scan of MAXI shown in Sections 3.2.1 and 3.2.2. The upper flux limits before and after the MAXI detection are given in Section 3.3, while the analysis and results for the Swift and SMARTS follow-up observations are presented in Section 3.4. The historical observations of this source are described in Section 3.5. In Section 4, we interpret the results obtained by MAXI, Swift and SMARTS and discuss the emission mechanism of the luminous soft X-ray transient detected by MAXI.

\section{OBSERVATION}

MAXI J0158-744 (Kimura et al. 2011) was first detected during a MAXI GSC scan (Figure 1(b)), centered at $T_{\text {trig }}+8 \mathrm{~s}$ within the $55 \mathrm{~s}$ triangular transit response (see Figure 9 in Sugizaki et al. 2011). It was subsequently detected twice by the MAXI SSC in scans at $+220 \mathrm{~s}$ and $+1296 \mathrm{~s}$ (Figure 1(c)). Hereafter, we designate the MAXI scans by the mid-time of the scan transit, referred to $T_{\text {trig. }}$. Subsequent GSC scans to date (up to 2013 July 8) have failed to detect the source. In addition, the source had not been detected in prior GSC scans, since MAXI observations started on 2009 August 14 up to the previous scan at $-5530 \mathrm{~s}$. The MAXI observations around $T_{\text {trig }}$ are summarized in Table 1.
Swift XRT performed follow-up observations from 0.44 days after $T_{\text {trig }}$ (see Table 1 of Li et al. 2012). Swift UVOT also observed the optical counterpart in image mode. Swift UVOT grism observations were performed on 2011 November 19 (+8.23 days after $T_{\text {trig }}$ ) and 2012 September 30 (324 days).

A ground-based optical spectrum, with relatively high resolution, was obtained on 2012 May 19 (190 days after the $T_{\text {trig }}$ ) with the Ritchey-Chrétien spectrograph ${ }^{19}$ on the SMARTS ${ }^{20} /$ CTIO $1.5 \mathrm{~m}$ telescope; this is a long slit spectrograph oriented eastwest (Walter et al. 2004, 2012). We used a 1 arcsec slit width and a Loral $1 \mathrm{~K} \mathrm{CCD}$ for the detector.

\section{ANALYSIS AND RESULTS \\ 3.1. Data Analysis of MAXI GSC}

On 2011 November 11, the position of MAXI J0158-744 was visible by three cameras of GSC-H (GSC_2, GSC_7 and GSC_8; Mihara et al. 2011; Sugizaki et al. 2011). One of these cameras (GSC_2) was operated at the nominal high voltage $(=1650 \mathrm{~V})$, while the other two (GSC_7 and GSC_8) were operated at the reduced voltage $(=1550 \mathrm{~V})$. We analyzed the GSC event data version 1.0 or later, which included the data taken by cameras operated at the nominal and reduced voltages. In these versions, the position and energy responses of the anodes 1 and 2 were significantly improved from the previous versions (0.x). We, therefore, used events taken from all anodes.

To make light curves within the interval of the scan-ID M+0 (Table 1), we followed the method shown in Morii et al. (2011a). Here, we selected events within $5 \mathrm{~mm}$ of the position coincident with this source along the anode wires, which corresponds to about $2^{\circ}$ on the sky. The obtained light curve data in energy bands of $2-4,4-10,10-20$, and $2-20 \mathrm{keV}$ were fitted with a model consisting of a triangular transit response curve for a point source with a constant flux and a constant background. The light curves are consistent with the model at the $90 \%$ confidence level, meaning that there was no significant variation of the source flux during that scan.

For the spectral analysis, we removed the GSC_8 data due to its poor response in the soft energy band. We selected a

\footnotetext{
19 http://www.ctio.noao.edu/spectrographs/60spec/60spec.html

20 The Small and Moderate Aperture Research Telescope System is a partnership that has overseen operations of four small telescopes at Cerro Tololo Interamerican Observatory since 2003.
} 


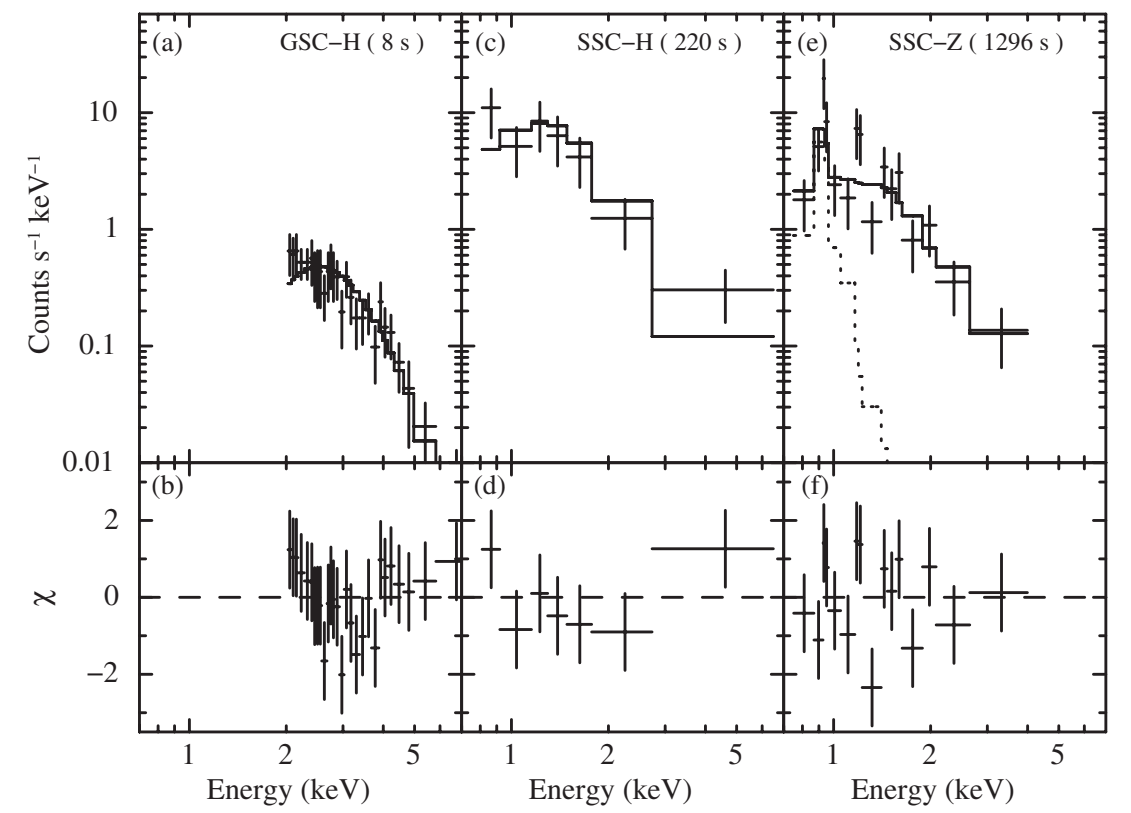

Figure 2. Energy spectra of MAXI J0158-744 observed by MAXI. Top left: Crosses are the GSC-H spectrum at the scan of $+8 \mathrm{~s}$. The histogram is the best-fit blackbody model (Table 2). Top middle: crosses are the SSC-H spectrum at the scan of $+220 \mathrm{~s}$. The histogram is the best-fit blackbody model (Table 2). Top right: crosses are the SSC-Z spectrum at the scan of $+1296 \mathrm{~s}$. The histogram is the best-fit blackbody + Mekal model with parameters shown in Section 3.2.2. The Mekal component is shown by a dotted histogram. All the spectra are plotted binned with a minimum of five counts per energy bin. Backgrounds are subtracted. Bottom: the residuals of the data from the models. Error bars, $1 \sigma$.

concentric circle and annulus centered at the target as the source and background regions, respectively. The radius of the source circle was set to 1.8 . The inner and outer radii of the background annulus were set to 2.2 and 3.5 , respectively. In both these regions, we excluded a circular region with a radius of 1.5 centered at a near-by bright X-ray source, SMC X-1 (Figure 1(b)). The spectrum and response files were made by the method described in Nakahira et al. (2012). The energy spectra obtained by the GSC Scan-ID M+0 are shown in Figure 2 (left). We rebinned the data with a minimum of 1 count per energy bin and applied Cash statistics (Cash 1979) in the fit. We used XSPEC v12.7.1 for the spectral analysis.

Since the location of this source is near the SMC, the interstellar absorption by the total Galactic H I column density toward this source, $N_{H}$, and optical extinction $E(B-V)$ are expected to be small. Thus, we fixed them for the following X-ray and optical spectral analysis. Two plausible different $N_{H}$ values are obtained from the HEASARC Web site: ${ }^{21} 1.36 \times$ $10^{21} \mathrm{~cm}^{-2}$ by using the Leiden/Argentine/Bonn (LAB) map (Kalberla et al. 2005) and $4.03 \times 10^{20} \mathrm{~cm}^{-2}$ by using the DL map (Dickey \& Lockman 1990). The corresponding optical extinctions $E(B-V)$ are 0.28 and $0.084 \mathrm{mag}$, respectively, derived by using the relation with the $\mathrm{H}$ I column density (Bohlin et al. 1978). On the other hand, the map of dust infrared emission (Schlegel et al. 1998) suggests $E(B-V)=0.050$, which is closer to that from the DL map. Therefore, we used the latter $N_{H}$ value, $4.03 \times 10^{20} \mathrm{~cm}^{-2}$, for the interstellar absorption. In the following analysis, unabsorbed flux is corrected only for the interstellar absorption.

We fit the GSC X-ray spectrum with absorbed blackbody, power-law, thermal bremsstrahlung, and Mekal (Mewe et al. 1985) models from $2.0-10.0 \mathrm{keV}$ with $N_{H}$ fixed to $4.03 \times$ $10^{20} \mathrm{~cm}^{-2}$; the results are shown in Table 2. The spectrum is statistically consistent with all the models. Adopting the

$\overline{21}$ http://heasarc.gsfc.nasa.gov/cgi-bin/Tools/w3nh/w3nh.pl
$N_{H}$ value of LAB map increases the unabsorbed flux by $2 \%$ from that using DL map. However, the difference in the spectral parameters and unabsorbed flux, when they are compared with the statistical uncertainty, are negligibly small.

\subsection{Data Analysis of MAXI SSC}

After the first detection of MAXI J0158-744 with the MAXI GSC scan at $+8 \mathrm{~s}$, MAXI SSC detected this source twice, as shown in Table 1. Before $+8 \mathrm{~s}$, MAXI J0158-744 was below the SSC detection limit of $\sim 200 \mathrm{mCrab}$ in each night scan. For the spectral analysis, we selected the source regions as shown in Figure 1(c) and reduced the SSC events in the same way as Kimura et al. (2013). While the second SSC detection at +1296 $\mathrm{s}$ was done at night in the orbit, the first SSC at $+220 \mathrm{~s}$ was done at the day-time. Since the SSC daytime data were contaminated by the visible and infrared light from the Sun (Tsunemi et al. 2010), we have to be careful of the analysis of the +220 s scan data. We estimated the area suffering from the contamination based on the event distribution, which led to the conclusion that $63 \%$ of the source area was not suitable for the spectral analysis. We thus used data from the remaining $37 \%$ area in the analysis.

The energy spectra obtained by the SSC scans at $+220 \mathrm{~s}$ and $+1296 \mathrm{~s}$ are shown in Figure 2 (middle, right). In the latter spectrum, since emission lines seemed to be present, we analyzed the SSC spectra by following the method shown in "low count spectra" of the XSPEC wiki site. ${ }^{22}$ To avoid losing information on emission lines as a result of the spectral binning, we did not bin the data and applied Cash statistics (Cash 1979) to the fits. We fit the source spectra with a model consisting of a source and a background component. Here, the background model was analytically described to approximate the actual background spectrum in $0.7-7.0 \mathrm{keV}$. It was constructed by averaging the two-year SSC data weighted with geomagnetic cut-off-rigidity, because the background events are caused by

\footnotetext{
$\overline{22}$ https://astrophysics.gsfc.nasa.gov/XSPEC wiki/low_count_spectra
} 
Table 2

Spectral Fitting Parameters with $1 \sigma$ Errors for the MAXI Scans

\begin{tabular}{|c|c|c|c|c|c|c|c|c|}
\hline Model $^{\text {a }}$ & $\Gamma^{\mathrm{b}}$ & $\begin{array}{l}k T^{\mathrm{c}} \\
(\mathrm{keV})\end{array}$ & $\begin{array}{c}R_{\mathrm{BB}}{ }^{\mathrm{d}} \\
\left(\times 10^{3} \mathrm{~km}\right)\end{array}$ & $\begin{array}{c}\mathrm{EM}^{\mathrm{e}} \\
\left(\times 10^{63} \mathrm{~cm}^{-3}\right)\end{array}$ & abund $^{f}$ & $\begin{array}{c}\text { Flux } \mathrm{g} \\
\left(\mathrm{erg} \mathrm{s}^{-1} \mathrm{~cm}^{-2}\right)\end{array}$ & $\begin{array}{l}\text { Luminosity } \\
\left(\mathrm{erg} \mathrm{s}^{-1}\right)\end{array}$ & $\begin{array}{l}\text { C-stat }{ }^{\mathrm{i}} \\
\left(\mathrm{DOF}^{\mathrm{j}}\right)\end{array}$ \\
\hline \multicolumn{9}{|c|}{ MAXI GSC-H (Scan-ID M+0, $+8 \mathrm{~s})$} \\
\hline $\mathrm{PL}$ & $4.89_{-0.28}^{+0.29}$ & $\cdots$ & $\cdots$ & $\cdots$ & $\cdots$ & $6.92_{-0.52}^{+0.55} \times 10^{-9}$ & $2.98_{-0.22}^{+0.24} \times 10^{39}$ & $43.4(60)$ \\
\hline BB & $\ldots$ & $0.45_{-0.03}^{+0.03}$ & $1.26_{-0.23}^{+0.29}$ & $\ldots$ & $\ldots$ & $6.74_{-1.01}^{+0.27} \times 10^{-9}$ & $2.90_{-0.43}^{+0.12} \times 10^{39}$ & $51.8(60)$ \\
\hline TB & $\ldots$ & $0.93_{-0.09}^{+0.10}$ & $\ldots$ & $5.9_{-1.5}^{+2.1}$ & $\ldots$ & $6.84_{-0.98}^{+0.31} \times 10^{-9}$ & $2.95_{-0.42}^{+0.13} \times 10^{39}$ & $45.3(60)$ \\
\hline Mekal & $\ldots$ & $0.94_{-0.11}^{+0.08}$ & $\ldots$ & $4.3_{-0.9}^{+1.6}$ & 0.1 (fix) & $6.80_{-0.67}^{+0.38} \times 10^{-9}$ & $2.93_{-0.29}^{+0.17} \times 10^{39}$ & $44.6(60)$ \\
\hline Mekal & $\cdots$ & $0.97_{-0.10}^{+0.11}$ & $\ldots$ & $2.4_{-2.1}^{+3.0}$ & $0.50_{-0.50}^{+5.74}$ & $6.74_{-5.14}^{+0.10} \times 10^{-9}$ & $2.90_{-2.21}^{+0.04} \times 10^{39}$ & $44.2(59)$ \\
\hline \multicolumn{9}{|c|}{ MAXI SSC-H (Scan-ID M+1, +220 s) } \\
\hline PL & $2.85_{-0.32}^{+0.34}$ & $\ldots$ & $\ldots$ & $\ldots$ & $\ldots$ & $4.96_{-0.84}^{+0.84} \times 10^{-8}$ & $2.13_{-0.36}^{+0.36} \times 10^{40}$ & $20.5(36)$ \\
\hline BB & $\ldots$ & $0.37_{-0.05}^{+0.05}$ & $4.8_{-1.2}^{+1.6}$ & $\ldots$ & $\ldots$ & $4.16_{-1.20}^{+0.47} \times 10^{-8}$ & $1.79_{-0.52}^{+0.20} \times 10^{40}$ & $30.2(36)$ \\
\hline TB & $\ldots$ & $1.46_{-0.35}^{+0.50}$ & $\ldots$ & $4.5_{-1.2}^{+1.7}$ & $\ldots$ & $4.71_{-1.13}^{+0.56} \times 10^{-8}$ & $2.03_{-0.49}^{+0.24} \times 10^{40}$ & $22.5(36)$ \\
\hline Mekal & $\ldots$ & $1.61_{-0.36}^{+0.48}$ & $\ldots$ & $3.4_{-0.6}^{+0.7}$ & 0.1 (fix) & $4.71_{-0.87}^{+0.64} \times 10^{-8}$ & $2.03_{-0.37}^{+0.28} \times 10^{40}$ & $24.0(36)$ \\
\hline Mekal & $\cdots$ & $1.51_{-0.41}^{+0.44}$ & $\cdots$ & $4.1_{-1.0}^{+1.5}$ & $<0.25(90 \%)$ & $4.77_{-0.83}^{+1.36} \times 10^{-8}$ & $2.05_{-0.36}^{+0.59} \times 10^{40}$ & $22.6(35)$ \\
\hline \multicolumn{9}{|c|}{ MAXI SSC-Z (Scan-ID M+2, +1296 s) } \\
\hline PL & $3.19_{-0.24}^{+0.26}$ & $\ldots$ & $\ldots$ & $\ldots$ & $\ldots$ & $1.71_{-0.18}^{+0.20} \times 10^{-8}$ & $7.37_{-0.78}^{+0.87} \times 10^{39}$ & $83.4(69)$ \\
\hline BB & $\ldots$ & $0.33_{-0.03}^{+0.03}$ & $2.29_{-0.39}^{+0.48}$ & $\ldots$ & $\ldots$ & $1.50_{-0.28}^{+0.10} \times 10^{-8}$ & $6.48_{-1.21}^{+0.45} \times 10^{39}$ & $84.2(69)$ \\
\hline TB & $\ldots$ & $0.94_{-0.14}^{+0.17}$ & $\ldots$ & $2.61_{-0.54}^{+0.71}$ & $\ldots$ & $1.61_{-0.30}^{+0.12} \times 10^{-8}$ & $6.91_{-1.30}^{+0.50} \times 10^{39}$ & $80.8(69)$ \\
\hline Mekal & $\ldots$ & $1.03_{-0.09}^{+0.10}$ & $\ldots$ & $1.42_{-0.16}^{+0.18}$ & 0.1 (fix) & $1.57_{-0.18}^{+0.17} \times 10^{-8}$ & $6.75_{-0.77}^{+0.73} \times 10^{39}$ & $80.8(69)$ \\
\hline Mekal & $\ldots$ & $0.96_{-0.10}^{+0.13}$ & $\ldots$ & $1.86_{-0.46}^{+0.57}$ & $0.041_{-0.031}^{+0.058}$ & $1.58_{-0.45}^{+0.11} \times 10^{-8}$ & $6.80_{-1.93}^{+0.49} \times 10^{39}$ & $79.4(68)$ \\
\hline
\end{tabular}

Notes.

${ }^{a}$ Absorbed power-law (PL), blackbody (BB), thermal bremsstrahlung (TB), and Mekal (Mewe et al. 1985) models are applied with $N_{H}$ fixed to $4.03 \times 10^{20} \mathrm{~cm}^{-2}$

(Section 3.1).

${ }^{\mathrm{b}}$ Photon index.

c Temperature.

${ }^{\mathrm{d}}$ Blackbody radius at the distance of $60 \mathrm{kpc}$.

${ }^{\mathrm{e}}$ Emission measure $\left(\mathrm{EM}=\int n_{e} n_{p} d V\right)$ at the distance of $60 \mathrm{kpc}$, where $n_{e}$ and $n_{p}$ are electron and proton number densities, respectively.

${ }^{\mathrm{f}}$ The abundance parameter (abund) of Mekal model in XSPEC equals to $10^{[X / H]}$, where $[X / H]$ is a metallicity of a metal $X$ (Mewe et al. 1985; Anders \& Grevesse 1989).

g Unabsorbed flux at 2.0-10.0 keV for GSC and 0.7-7.0 keV for SSC.

${ }^{\mathrm{h}}$ Luminosity at $2.0-10.0 \mathrm{keV}$ for GSC and $0.7-7.0 \mathrm{keV}$ for SSC at the distance of $60 \mathrm{kpc}$.

${ }^{\mathrm{i}}$ Cash statistics (Cash 1979) calculated by binning the data with a minimum of 1 count per energy bin.

${ }^{\mathrm{j}}$ Degrees of freedom.

charged particles and cosmic diffuse X-rays. The details of the background spectrum are shown in Kimura et al. (2013). We fit the spectra by absorbed blackbody, power-law, thermal bremsstrahlung, and Mekal (Mewe et al. 1985) models in $0.7-7.0 \mathrm{keV}$ with $N_{H}$ fixed to $4.03 \times 10^{20} \mathrm{~cm}^{-2}$ (Section 3.1); the results are shown in Table 2. For the Mekal model, we let the abundance parameter (hereafter, abund. $)^{23}$ be free or fixed to 0.1 (a typical abundance of the SMC; Carrera et al. 2008). For the first SSC spectrum (+220 s), the data are statistically consistent with all the models, While, for the second, the free abundance Mekal model is preferred. Adopting the $N_{H}$ value of LAB map increases the unabsorbed fluxes by up to $20 \%$ from those using DL map. However, differences in all the spectral parameters and unabsorbed fluxes are not significant (less than $2.6 \sigma$ level of the statistical uncertainty).

As shown in Table 2, the initial X-ray outburst of MAXI J0158-744 detected by MAXI GSC and SSC was peaked at $+220 \mathrm{~s}$. The peak luminosity was extraordinarily luminous, $2 \times 10^{40} \mathrm{erg} \mathrm{s}^{-1}$ in $0.7-7.0 \mathrm{keV}$, which is two orders of magnitude

23 The abundance parameter (abund) of Mekal model in XSPEC equals to $10^{[X / H]}$. Here, $[X / H]=\log _{10}\left(n_{\mathrm{X}} / n_{\mathrm{H}}\right)_{\text {source }}-\log _{10}\left(n_{\mathrm{X}} / n_{\mathrm{H}}\right)_{\text {sun }}$ is a metallicity of a metal $X$, where $\left(n_{\mathrm{X}}\right)_{\mathrm{Y}}$ represents the number density of an element $(X)$ in a source $(Y)$. larger than the Eddington luminosity of a solar mass object. In the following subsections, we investigate the MAXI SSC spectrum at $+1296 \mathrm{~s}$, where emission lines are apparent, with two scenarios: shock-induced emission and photospheric emission at the fireball phase (see Section 4).

\subsubsection{Detailed Spectral Analysis of MAXI SSC at $+1296 \mathrm{~s}$ with a Shock-induced Emission Model}

To investigate the emission lines in the spectrum at $+1296 \mathrm{~s}$, we first fit the spectrum with models consisting of thermal bremsstrahlung continuum and Gaussian lines, whose widths were fixed to be small against the detector energy resolution, $85 \mathrm{eV}$ (FWHM) at $1.0 \mathrm{keV}$ (Kimura et al. 2013). The best-fit parameters are summarized in Table 3 (upper) and the models are shown in Figure 3 ((a), (b), (c), (d)). The results of the likelihood ratio tests (Cash 1979), in the last two rows of Table 3 (upper), indicate that the addition of the Gaussian lines at the energies $E_{1}, E_{2}$ and $E_{3}$ one by one improves the fits with a chance probability of $0.0044,0.084$, and 0.085 , respectively. The line at the energy $E_{1}$ is the most significant, and is inferred as a resonance line of He-like neon $(0.922 \mathrm{keV})$. The other two lines are less significant than $2 \sigma$ and no corresponding major lines exist at these energies. However, the line center energy 
Table 3

Spectral Fitting Parameters of a Continuum + Emission Lines for the SSC-Z Scan at +1296 s and the Likelihood Ratio Test

\begin{tabular}{|c|c|c|c|c|}
\hline Model & $\mathrm{TB}^{\mathrm{a}}$ & $\mathrm{TB}+$ Line $^{\mathrm{b}}$ & $\mathrm{TB}+2$ Lines & $\mathrm{TB}+3$ Lines \\
\hline$k T^{\mathrm{c}}(\mathrm{keV})$ & $0.94_{-0.14}^{+0.17}$ & $1.12_{-0.19}^{+0.25}$ & $1.21_{-0.20}^{+0.34}$ & $1.28_{-0.25}^{+0.36}$ \\
\hline $\operatorname{EM}^{\mathrm{d}}\left(\times 10^{63} \mathrm{~cm}^{-3}\right)$ & $2.61_{-0.54}^{+0.71}$ & $1.78_{-0.40}^{+0.53}$ & $1.42_{-0.37}^{+0.44}$ & $1.17_{-0.32}^{+0.43}$ \\
\hline$E_{1}{ }^{\mathrm{e}}(\mathrm{keV})$ & $\ldots$ & $0.93_{-0.01}^{+0.01}$ & $0.93_{-0.01}^{+0.01}$ & $0.93_{-0.01}^{+0.01}$ \\
\hline $\mathrm{EW}_{1}{ }^{\mathrm{f}}(\mathrm{keV})$ & $\ldots$ & $0.19_{-0.07}^{+0.13}$ & $0.26_{-0.10}^{+0.22}$ & $0.33_{-0.11}^{+0.26}$ \\
\hline$E_{2}{ }^{\mathrm{g}}(\mathrm{keV})$ & $\ldots$ & $\ldots$ & $1.19_{-0.02}^{+0.02}$ & $1.19_{-0.02}^{+0.02}$ \\
\hline $\mathrm{EW}_{2}(\mathrm{keV})$ & $\ldots$ & $\ldots$ & $0.14_{-0.08}^{+0.14}$ & $0.18_{-0.08}^{+0.20}$ \\
\hline$E_{3}{ }^{\mathrm{h}}(\mathrm{keV})$ & $\ldots$ & $\ldots$ & $\ldots$ & $1.57_{-0.04}^{+0.03}$ \\
\hline $\mathrm{EW}_{3}(\mathrm{keV})$ & $\ldots$ & $\ldots$ & $\ldots$ & $0.24_{-0.14}^{+0.26}$ \\
\hline$C-\operatorname{stat}^{\mathrm{i}}\left(\mathrm{DOF}^{\mathrm{j}}\right)$ & $342.9(1723)$ & $332.1(1721)$ & 327.1(1719) & $322.2(1717)$ \\
\hline$\overline{\Delta C(\Delta \mathrm{DOF})^{\mathrm{k}}}$ & $\ldots$ & $10.8(2)$ & $5.0(2)$ & $4.9(2)$ \\
\hline$P$-value ${ }^{l}$ & $\ldots$ & 0.0044 & 0.084 & 0.085 \\
\hline Model & $\mathrm{BB}^{\mathrm{m}}$ & BB + Line & $\mathrm{BB}+2$ Lines & BB + 3 Lines \\
\hline$\overline{k T^{\mathrm{c}}(\mathrm{keV})}$ & $0.33_{-0.03}^{+0.03}$ & $0.38_{-0.03}^{+0.04}$ & $0.41_{-0.04}^{+0.04}$ & $0.42_{-0.04}^{+0.05}$ \\
\hline$R_{\mathrm{BB}}{ }^{\mathrm{n}}\left(\times 10^{3} \mathrm{~km}\right)$ & $2.29_{-0.39}^{+0.48}$ & $1.54_{-0.28}^{+0.35}$ & $1.22_{-0.23}^{+0.35}$ & $1.10_{-0.22}^{+0.35}$ \\
\hline$E_{1}{ }^{\mathrm{e}}(\mathrm{keV})$ & $\ldots$ & $0.93_{-0.01}^{+0.01}$ & $0.93_{-0.01}^{+0.01}$ & $0.93_{-0.01}^{+0.01}$ \\
\hline $\mathrm{EW}_{1}{ }^{\mathrm{f}}(\mathrm{keV})$ & $\ldots$ & $0.32_{-0.11}^{+0.21}$ & $0.45_{-0.14}^{+0.36}$ & $0.55_{-0.18}^{+0.45}$ \\
\hline$E_{2}{ }^{\mathrm{g}}(\mathrm{keV})$ & $\ldots$ & $\ldots$ & $1.19_{-0.02}^{+0.02}$ & $1.19_{-0.02}^{+0.02}$ \\
\hline $\mathrm{EW}_{2}(\mathrm{keV})$ & $\ldots$ & $\ldots$ & $0.16_{-0.08}^{+0.16}$ & $0.20_{-0.09}^{+0.21}$ \\
\hline$E_{3}{ }^{\mathrm{h}}(\mathrm{keV})$ & $\ldots$ & $\ldots$ & $\ldots$ & $1.57_{-0.05}^{+0.04}$ \\
\hline $\mathrm{EW}_{3}(\mathrm{keV})$ & $\ldots$ & $\ldots$ & $\ldots$ & $0.16_{-0.11}^{+0.22}$ \\
\hline$C$-stat $^{\mathrm{i}}\left(\mathrm{DOF}^{\mathrm{j}}\right)$ & $343.8(1723)$ & $326.7(1721)$ & 321.1(1719) & $318.2(1717)$ \\
\hline$\overline{\Delta C(\Delta \mathrm{DOF})^{\mathrm{k}}}$ & $\ldots$ & 17.1(2) & $5.6(2)$ & $2.9(2)$ \\
\hline$P$-value ${ }^{l}$ & $\ldots$ & $1.9 \times 10^{-4}$ & 0.061 & 0.23 \\
\hline
\end{tabular}

Notes.

${ }^{a}$ Thermal bremsstrahlung.

${ }^{\mathrm{b}}$ Gaussian line.

c Temperature.

${ }^{\mathrm{d}}$ Emission measure.

${ }^{\mathrm{e}}$ Line energy $\left(E_{1}\right)$ is constrained between 0.7 and $1.1 \mathrm{keV}$.

${ }^{\mathrm{f}}$ Equivalent width.

${ }^{\mathrm{g}} E_{2}$ : between 1.1 and $1.3 \mathrm{keV}$.

${ }^{\mathrm{h}} E_{3}$ : between 1.3 and $1.7 \mathrm{keV}$.

${ }^{\mathrm{i}}$ Cash statistics (Cash 1979) without binning.

${ }^{\mathrm{j}}$ Degrees of freedom.

${ }^{\mathrm{k}}$ The difference of the $C$-stat (DOF) between this column and the next column to the left.

${ }^{1} \Delta C$ is distributed as $\chi^{2}$ with degrees of freedom of $\Delta \mathrm{DOF}$ (Cash 1979).

m Blackbody.

${ }^{\mathrm{n}}$ Blackbody radius at the distance of $60 \mathrm{kpc}$.

$E_{2}$ may suggest a radiative recombination continuum of He-like neon $(1.20 \mathrm{keV})$ or Lyman beta line of H-like neon $(1.24 \mathrm{keV})$, and the $E_{3}$ may suggest a resonance line of He-like aluminum $(1.60 \mathrm{keV})$ or $1 s 3 p^{1} P_{1} \rightarrow 1 s^{2}{ }^{1} S_{0}$ line of He-like magnesium $(1.58 \mathrm{keV})$. The neon emission line suggests that the initial bright outburst would have been produced by an optically-thin thermal emission mechanism, that was located in a region heated by the shock wave of a nova explosion, as seen in some novae (RS Ophiuchi and V407 Cyg; Sokoloski et al. 2006; Nelson et al. 2012).

We next tried to fit the spectrum with more physically motivated models. Although it can be fit with an optically thin thermal emission model (Mekal in XSPEC terminology) with a temperature of $\sim 1.0 \mathrm{keV}$ (Table 2 ), the model cannot produce the observed strong He-like neon line. To reproduce this line, another optically-thin thermal component with a lower temperature (about $0.1 \mathrm{keV}$ ) is necessary. Thus, we examined a model consisting of two Mekal models $\left(\right.$ Mekal $_{\mathrm{LT}}+$ Mekal $\left._{\mathrm{HT}}\right)$, that have temperatures at $\sim 0.1 \mathrm{keV}$ in the lower component (LT) and $\sim 1.0 \mathrm{keV}$ in the higher component (HT), respectively. The best-fit result in Table 2 shows that the abund of the Mekal is consistent with that of the SMC. This conclusion, however, is not completely correct, because the fit included an energy range affected by the He-like neon line produced by the Mekal ${ }_{\text {LT }}$ component. To determine the abund of $\mathrm{Mekal}_{\mathrm{HT}}$, we fit the spectrum excluding the energy range $0.8-1.0 \mathrm{keV}$, and then obtained an upper limit for the abund of 0.25 (90\% confidence limit), which is consistent with that of the SMC (Carrera et al. 2008). We thus decided to fix abund of the Mekal ${ }_{\mathrm{HT}}$ component to 0.1 . For the $\mathrm{Mekal}_{\mathrm{LT}}$ component, the observed strong He-like neon line suggests a large abundance for neon. We postulate that the Mekal LT $_{\text {T }}$ component was produced in a reverse shocked region that had material that was ejecta from the nova explosion (see Section 4). 

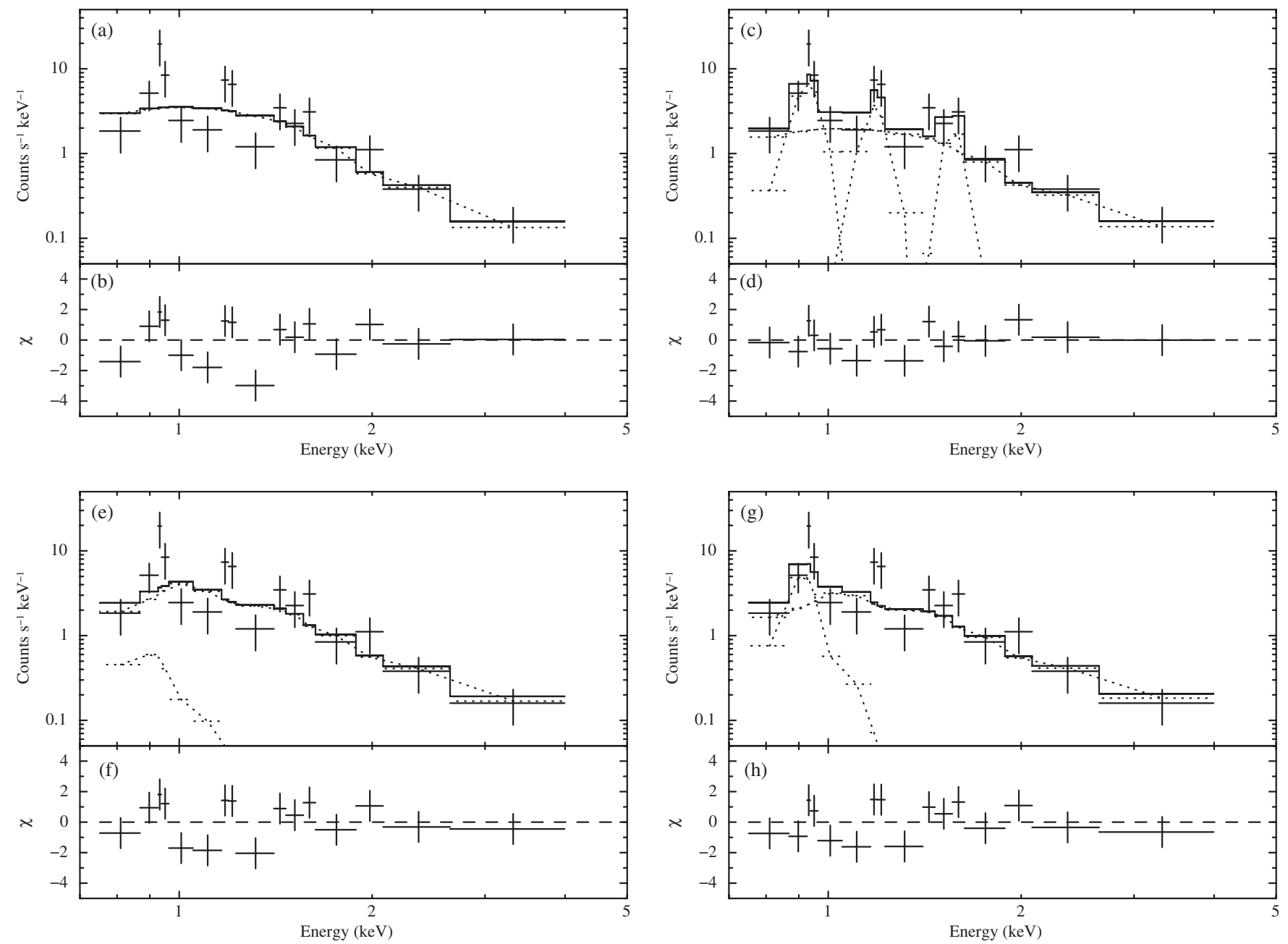

Figure 3. MAXI SSC-Z spectrum of MAXI J0158-744 at +1296 s. (a, c) The histograms are the best-fit thermal bremsstrahlung (a) and thermal bremsstrahlung +3 Gaussian lines models (c). (e) The histogram is the best-fit Mekal $L_{\text {LT }}+$ Mekal $_{\mathrm{HT}}$ model, where the abunds of Ne and the other elements in the Mekal ${ }_{\mathrm{LT}}$ component are set to 0.1 and 0.1, respectively (Table 4, the first row). (g) The histogram is the same model as (e), where these abunds are set to 10.0 and 0.1 (Table 4 , the fifth row). All the spectra are plotted binned with a minimum of 5 counts per energy bin. (b, d, f, h) The residuals of the data from the models. Error bars, $1 \sigma$.

To estimate the abunds of the Mekal ${ }_{\mathrm{LT}}$ component, we fit the spectrum with the Mekal ${ }_{\mathrm{LT}}+\mathrm{Mekal}_{\mathrm{HT}}$ model, assuming six combinations of the abunds of neon and the other elements for $\mathrm{Mekal}_{\mathrm{LT}}$ as shown in Table 4. Here, at the first step in the spectral fit, we let the temperature and emission measure of the Mekal $_{\text {HT }}$ component freely vary. When the $1 \sigma$ error range of the temperature of the Mekal ${ }_{\text {LT }}$ was not constrained to less than $0.3 \mathrm{keV}$ in the first step (the first three cases of Table 4), we fixed the temperature of $\mathrm{Mekal}_{\mathrm{HT}}$ and then the emission measure of Mekal ${ }_{\text {HT }}$ to the best-fit values. These best-fit values were obtained by fitting the same spectrum, excluding the energy range $0.8-1.0 \mathrm{keV}$, with a single Mekal component and the abund fixed to 0.1 . For the first case of Table 4, the $1 \sigma$ error range of the Mekal ${ }_{\text {LT }}$ could not be constrained to be less than $0.3 \mathrm{keV}$, even after both the temperature and emission measure of Mekal ${ }_{\text {HT }}$ were fixed. Figure 3 (e), (f), (g), (h) presents the difference in these spectral fits with respect to the neon abunds of the Mekal ${ }_{L T}$ component. As a result, the neon abund of the Mekal ${ }_{\text {LT }}$ was suggested to be much higher than that of the SMC (Table 4), which indicates that the Mekal ${ }_{\mathrm{LT}}$ component originates in ejecta from the nova. The unabsorbed flux in 0.7-7.0 keV, assuming the $\mathrm{Mekal}_{\mathrm{LT}}+\mathrm{Mekal}_{\mathrm{HT}}$ model with parameters shown in the fifth row of Table 4 (the best-fit model), is $1.63_{-0.17}^{+0.19} \times 10^{-8} \mathrm{erg} \mathrm{s}^{-1} \mathrm{~cm}^{-2}$.

\subsubsection{Detailed Spectral Analysis of MAXI SSC at $+1296 s$ with Photospheric Emission at the Fireball Phase}

The initial bright outburst detected by MAXI may also be explained by photospheric emission at the ignition phase of a nova explosion, the so-called fireball phase (see Section 4). In this scenario, the main continuum component in the spectrum of MAXI SSC at $+1296 \mathrm{~s}$ is blackbody emission, while the emission lines come from the optically thin region surrounding the photosphere (see Figure 5 in Section 4). Thus, we fit the spectrum with models consisting of blackbody continuum and Gaussian lines. The results of the fit are shown in Table 3 (lower). The addition of the Gaussian line at the energy $E_{1}$ significantly improves the fits with a chance probability of $1.9 \times 10^{-4}$, while the other two lines are detected at $<2 \sigma$. The identifications of these lines are the same as in Section 3.2.1. The detection of the neon emission line suggests that the spectrum contains an optically-thin thermal emission component. Therefore, this spectrum could be explained by a composite model of a blackbody and a Mekal with a temperature below $0.3 \mathrm{keV}$, and an exceptionally large neon abundance, similar to the two Mekal models in Section 3.2.1. When the abunds of neon and the other elements are set to 10 and 0.1 , respectively, the resultant best-fit spectral parameters are as follows: the 
Table 4

Spectral Fitting Parameters of the Mekal $_{\mathrm{LT}}{ }^{\mathrm{a}}+\mathrm{Mekal}_{\mathrm{HT}}{ }^{\mathrm{b}}$ Model for the SSC-Z Scan at $+1296 \mathrm{~s}$ with $1 \sigma$ Errors

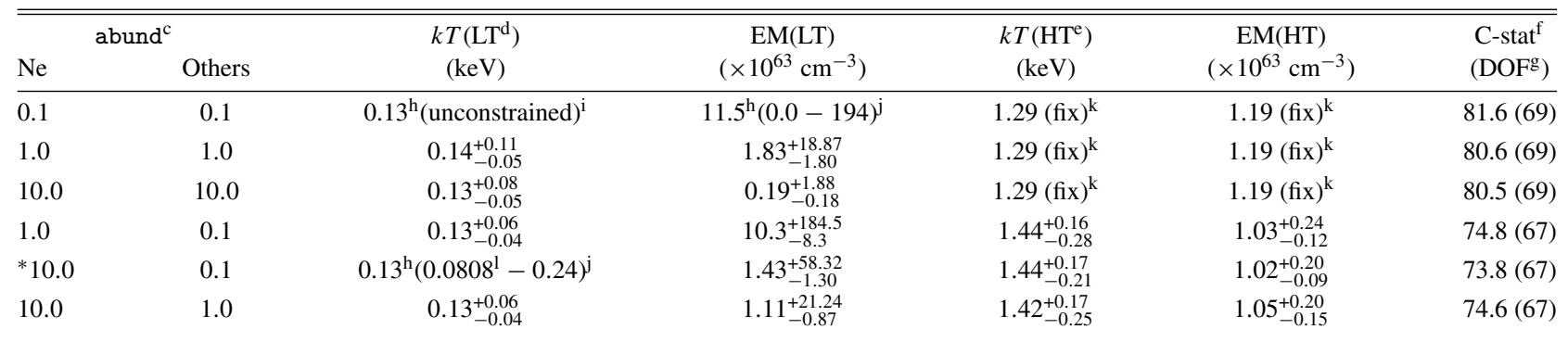

Notes.

a To set different abunds for neon and the other elements, vmekal model in XSPEC was used for the Mekal $\mathrm{LT}_{\text {component. }}$

$\mathrm{b}$ The abund of Mekal $\mathrm{HT}$ was fixed to 0.1 .

c The abunds of the Mekal ${ }_{\text {LT }}$ component.

${ }^{\mathrm{d}}$ LT: Lower temperature.

e HT: Higher temperature.

${ }^{\mathrm{f}}$ Cash statistics (Cash 1979) calculated by binning the data with a minimum of 1 count per energy bin.

$\mathrm{g}$ Degrees of freedom.

${ }^{\mathrm{h}}$ Best-fit value.

i $1 \sigma$ error interval is not constrained between $0.0808 \mathrm{keV}^{\mathrm{l}}$ and $0.3 \mathrm{keV}$.

j $1 \sigma$ error interval.

${ }^{\mathrm{k}}$ The best-fit temperature and emission measure obtained by a single Mekal fits for the same spectrum excluding the energy range $0.8-1.0$ keV with the abund fixed to 0.1 .

${ }^{1}$ Computational lower boundary of the Mekal model.

* The best fit and preferred model.

temperature and emission measure of the Mekal component are $0.14(0.0808-0.28) \mathrm{keV}$ (see footnotes $\mathrm{h}, \mathrm{j}$, and 1 of Table 4) and $1.13_{-1.03}^{+62.97} \times 10^{63} \mathrm{~cm}^{-3}$, respectively. The temperature and radius of the blackbody component are $0.39_{-0.04}^{+0.04} \mathrm{keV}$ and $1.47_{-0.31}^{+0.37} \times 10^{3} \mathrm{~km}$, respectively.

\subsection{Upper Limits on Other MAXI GSC Scans}

In the scans at $-5530 \mathrm{~s}$ and $+5545 \mathrm{~s}$ (Table 1), MAXI J0158-744 was not detected by MAXI GSC. To calculate the upper limits on these fluxes, we assumed the best-fit Mekal $_{\mathrm{LT}}+$ Mekal $_{\text {HT }}$ model obtained by the MAXI SSC scan at $+1296 \mathrm{~s}$ (Section 3.2.1) and the best-fit blackbody model (the ScanID $M+1$ in Table 2). In the former model, the abund of the Mekal $_{\text {HT }}$ and the Mekal ${ }_{\text {LT }}$ was fixed to 0.1, except for the neon abund in the Mekal $_{\text {LT }}$ fixed to 10 (Table 4, the fifth row). The $90 \%$ confidence level upper limits on the unabsorbed flux in $0.7-7.0 \mathrm{keV}$ for these scans are $<1.94 \times 10^{-9}$ and $<8.29 \times 10^{-10} \mathrm{erg} \mathrm{s}^{-1} \mathrm{~cm}^{-2}$ in the two Mekal model, and $<2.75 \times 10^{-9}$ and $<9.60 \times 10^{-10} \mathrm{erg} \mathrm{s}^{-1} \mathrm{~cm}^{-2}$ in the blackbody model.

In addition, in all five scans between the scan at +5545 s (+0.064 days) and the start of the Swift XRT follow-up (i.e., at $+0.128,+0.192,+0.256,+0.320$, and +0.385 days), MAXI J0158-744 was not detected by MAXI GSC. The $90 \%$ confidence-level upper limit on the unabsorbed flux in the $0.7-7.0 \mathrm{keV}$ band for this period was $3.5 \times 10^{-10} \mathrm{erg} \mathrm{s}^{-1} \mathrm{~cm}^{-2}$, assuming the former model and $2.5 \times 10^{-10} \mathrm{erg} \mathrm{s}^{-1} \mathrm{~cm}^{-2}$ for the latter model.

\subsection{Analysis of Follow-up Observations \\ 3.4.1. Analysis of Swift Observations}

We analyzed the same Swift XRT archival data as listed in Table 1 of Li et al. (2012), using Swift software version 3.9, released as part of HEASOFT 6.12. We extracted the source events from a circle with optimal radii $\left(47^{\prime \prime}-23^{\prime \prime}\right)$ and the background from an offset circular region of radius $142^{\prime \prime}$. For the data on day 0.54 , we excluded events from the inner $5^{\prime \prime}$ of the point-spread function (PSF) to avoid pile-up. In the spectral fit, we used the redistribution matrix files of swxpc0to12s6_20010101v013.rmf in Photon Counting (PC) mode and swxwt0to2s6_20010101v014.rmf in Windowed Timing (WT) mode. The ancillary response files were generated by using the commands xrtexpomap (to create the exposure maps) and xrtmkarf.

We fit the Swift XRT spectra with absorbed blackbody or Mekal models with the intrinsic column $N_{H}$ allowed to vary. These models include two absorption components: the interstellar absorption $N_{H}$ fixed at $4.03 \times 10^{20} \mathrm{~cm}^{-2}$ (Section 3.1) and intrinsic absorption. In the Mekal model, the abund was fixed to 0.1, a typical abund of the SMC (Carrera et al. 2008; see also Section 3.2.1). The unabsorbed flux obtained by the blackbody fits are shown in Figure 4.

We also analyzed the Swift UVOT data obtained at the same time as Swift XRT, using the Swift software version 3.9, released as part of HEASOFT 6.12. The image data of each filter, from each observation sequence, i.e., with a given observation ID, were summed using uvotimsum. However, for images taken within two days of the outburst $(b, u$, and $w 1$ bands) individual exposures were long enough that summing was not necessary. Photometry of the source in individual sequences was derived via uvotmaghist, using an extraction region of radius $5^{\prime \prime}$ and a suitable background region. Magnitudes are based on the UVOT photometric system (Poole et al. 2008). XSPEC compatible spectral files for the source were created using the same region with uvot2pha.

The $u$ band light curve is shown in Figure 4. We calculated the absolute magnitude of the enhanced emission after extinction correction, where $E(B-V)=0.050 \mathrm{mag}$ (Section 3.1), $A_{V} / E(B-V)=3.1, A_{u}=1.664 A_{V}$ (Schlegel et al. 1998), 


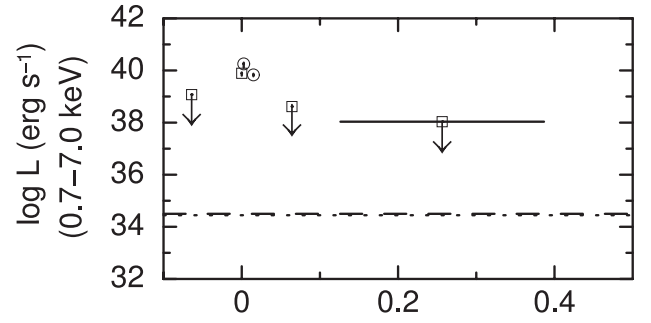

Time (days) relative to Trigger Time

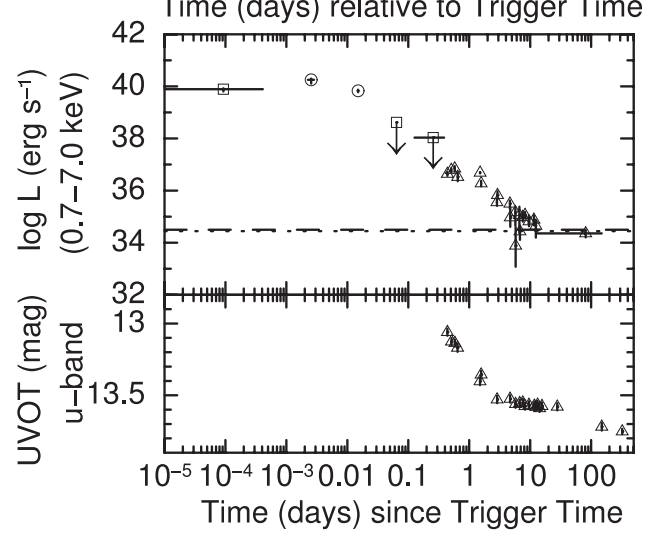

Figure 4. Evolution of the fluxes of MAXI J0158-744. Top, Middle: the X-ray luminosity with horizontal axes in linear (top) and log (middle) scales. Here, the source distance is assumed to be the SMC distance of $60 \mathrm{kpc}$. Bottom: the $u$ band magnitude. The squares, circles and triangles show the data obtained by MAXI GSC (Sections 3.1 and 3.3), MAXI SSC (Sections 3.2 and 3.2.2), and Swift (Section 3.4), respectively. For the MAXI data, the luminosities converted from the fluxes of Table 1 and Section 3.3 are shown. For the Swift XRT data, those obtained by blackbody fits are shown (Section 3.4). The vertical error bars represent $1 \sigma$ level. Horizontal dashed and dotted lines show the $90 \%$ confidence upper limits obtained by XMM-Newton slew survey and ROSAT PSPC, respectively, assuming SSS Model (Section 3.5).

and the SMC distance of $d=60 \mathrm{kpc}$ were assumed. Here, we subtracted the flux in the plateau phase $(13.58 \mathrm{mag}$, average of $u$-band magnitudes from 11.65 days to 27.86 days.). By fitting the light curve of the enhanced emission with a linear function, we obtained the absolute magnitude of $-5.04 \pm 0.07$ mag at 0.44 days and the speed class indicator parameter defined by the time to decline $2 \mathrm{mag}$ from maximum (Warner 2008), $t_{d, 2}=1.9 \pm 0.2(1 \sigma)$ days. This classifies the event as a "very fast nova" (Warner 2008), assuming that the optical enhancement was due to the photospheric emission as in usual novae.

To investigate the optical enhanced emission, we made a difference spectrum from the Swift UVOT photometry over the six filter bandpasses ${ }^{24}$ between day 1.5 and 149 , and we fit it with a blackbody model with fixed interstellar extinction (Section 3.1). The blackbody temperature of $1.22_{-0.10}^{+0.11} \times 10^{4} \mathrm{~K}$ and the radius of $6.58_{-0.89}^{+1.03} \times 10^{11} \mathrm{~cm}$ were obtained at the best fit. We also fit the spectrum from day 149 with the blackbody model, obtaining a temperature of $2.34_{-0.04}^{+0.04} \times 10^{4} \mathrm{~K}$ and a radius of $5.81_{-0.14}^{+0.14} \times 10^{11} \mathrm{~cm}$. The extrapolation of the bestfit blackbody spectrum toward the UV region is consistent with the UV flux obtained by the Galaxy Evolution Explorer (Morrissey et al. 2005) during the pre-outburst phase (Li et al. 2012). In addition, Li et al. (2012) reported that the $I$-band flux returned to the pre-outburst level $\sim 60$ days after $T_{\text {trig. }}$. So, we can assume that the flux at day 149 contains only emission from the binary companion star. The obtained temperature and radius

\footnotetext{
24 Central wavelengths (FWHM) in angstroms (Poole et al. 2008): $v: 5468$ (769), $b: 4392$ (975), $u$ : 3465 (785), uvw1: 2600 (693), uvm2: 2246 (498), uvw2: 1928 (657)
}

are consistent with those of a B-type star, as shown in Li et al. (2012).

Swift UVOT grism spectra are close to that of an early B-type star. No clear emission lines can be identified above the noise, as shown in Li et al. (2012).

\subsubsection{Ground-based Optical Spectroscopy by SMARTS}

We obtained three $200 \mathrm{~s}$ spectra of the optical counterpart of MAXI J0158-744 to filter for cosmic rays. We combine the three images, and extract the spectrum by fitting a Gaussian in the spatial direction at each pixel. Wavelength calibration is accomplished by fitting a $3^{\text {rd }}$ to $6^{\text {th }}$ order polynomial to the calibration lamp line positions. The optical spectrum covers nearly the entire optical band (3300-9500 A) at $17 \AA$ resolution.

There are clear emission lines of $H_{\alpha}$ and $H_{\beta}$, with equivalent widths of $16 \AA$ and $1 \AA$, respectively. We could not find any other significant emission or absorption lines above the noise level. The SMARTS spectrum matches the New Technology Telescope (NTT) spectrum of Li et al. (2012), albeit with a worse signal-to-noise ratio.

\subsection{Historical X-Ray Fluxes}

To investigate the activity of MAXI J0158-744 before the discovery, we searched for previous X-ray observations of the area including the target position. This region was observed by the ROSAT all-sky survey, XMM-Newton slew survey, and MAXI GSC. The source was undetected in all these observations. We calculated the upper limits on the unabsorbed fluxes in an energy range of $0.7-7.0 \mathrm{keV}$, assuming the bestfit Mekal $_{\mathrm{LT}}+$ Mekal $_{\mathrm{HT}}$ model (Table 4, the fifth row; Outburst Model) and a typical spectrum in the SSS phase observed by Swift XRT, an absorbed blackbody with a temperature of $0.1 \mathrm{keV}$ (SSS Model; Li et al. 2012).

The ROSAT all-sky survey covered this field, with an exposure of $775 \mathrm{~s}$ in total between 1990 September 22 and December 3. These data provide a PSPC count rate upper limit of 0.14 counts $\mathrm{s}^{-1}$ (90\% confidence limit) over $0.1-2.5 \mathrm{keV}$; corresponding to $<2.1 \times 10^{-13}$ and $<6.5 \times 10^{-14} \mathrm{erg} \mathrm{cm}^{-2} \mathrm{~s}^{-1}(0.7-7.0 \mathrm{keV})$, assuming Outburst Model and SSS Model, respectively (Table 5).

XMM-Newton slewed over the source three times on 2006 November, 2007 October, and 2009 November. We obtained EPIC pn count rate upper limits of $0.35,1.5$ and 0.50 counts $\mathrm{s}^{-1}(2 \sigma$ level) in $0.2-12 \mathrm{keV}$, respectively. The corresponding unabsorbed fluxes $(0.7-7.0 \mathrm{keV})$ are shown in Table 5 .

We also analyzed the MAXI GSC image in the $4-10 \mathrm{keV}$ band integrated for seven months from 2009 September 1 to 2010 March 31. Applying the same analysis procedure as Hiroi et al. (2011) use, we obtain a 90\% confidence-level upper limit of $0.10 \mathrm{mCrab}$. This corresponds to an unabsorbed flux of $<1.8 \times 10^{-11} \mathrm{erg} \mathrm{cm}^{-2} \mathrm{~s}^{-1}(0.7-7.0 \mathrm{keV})$, assuming Outburst Model.

\section{DISCUSSION}

The X-ray transient MAXI J0158-744 is characterized by (1) a soft X-ray spectrum with most of the X-ray photons being detected below $4 \mathrm{keV}$ (Figures 1 and 2), (2) a short duration (between $t_{2}-t_{1}=1.3 \times 10^{3} \mathrm{~s}$ and $t_{3}-t_{-1}=1.10 \times 10^{4} \mathrm{~s}$; Table 1), (3) a very rapid rise time $\left(<t_{1}-t_{-1}=5.5 \times 10^{3} \mathrm{~s}\right.$; Table 1), and (4) a huge peak luminosity of $2 \times 10^{40} \mathrm{erg} \mathrm{s}^{-1}$ in the $0.7-7.0 \mathrm{keV}$ band recorded at the second MAXI scan. The unusually soft spectrum of the outburst is beyond astronomers' expectations, because most short-lived luminous transient 
Table 5

Summary of ROSAT All-sky Survey and XMM-Newton Slew Survey Observations

\begin{tabular}{lcccc}
\hline \hline Date & Telescope & Exp.(s) $^{\mathrm{a}}$ & ${\text { Flux }(\text { Outburst })^{\mathrm{b}}}$ & ${\text { Flux }(\text { SSS })^{\mathrm{b}}}^{\text {1990-09-22-1990-12-03 }}$ \\
ROSAT & 775 & $<2.1 \times 10^{-13}$ & $<6.5 \times 10^{-14}$ \\
2006-11-01 10:03:35 & XMM-Newton & 8.9 & $<3.6 \times 10^{-13}$ & $<7.3 \times 10^{-14}$ \\
2007-10-28 11:57:17 & XMM-Newton & 2.1 & $<1.6 \times 10^{-12}$ & $<3.2 \times 10^{-13}$ \\
2009-11-30 23:41:46 & XMM-Newton & 6.1 & $<5.2 \times 10^{-13}$ & $<1.1 \times 10^{-13}$ \\
\hline
\end{tabular}

Notes.

${ }^{\text {a }}$ Exposure in units of seconds. For XMM-Newton, it is corrected for vignetting to the on-axis equivalent value.

b The upper limit on the unabsorbed flux in a unit of $\operatorname{erg~s}^{-1} \mathrm{~cm}^{-2}$ in an energy range of 0.7-7.0 keV, where Outburst and SSS Models are assumed (see text). The 90\% confidence level for ROSAT and $2 \sigma$ level for XMM-Newton.

X-ray sources are hard X-ray emitters, e.g., gamma-ray bursts (Band et al. 1993), soft gamma repeaters (Woods \& Thompson 2006), super-giant fast X-ray transients (Sguera et al. 2006), and X-ray bursts (Galloway et al. 2008). The discovery of MAXI J0158-744, together with supernova shock breakouts (Soderberg et al. 2008), suggests that the wide-field monitoring experiments in soft $\mathrm{X}$-rays $(<2 \mathrm{keV})$ will open new discovery fields.

Li et al. (2012) reported the optical spectra obtained by SAAO and ESO, showing that the source is a Be star. In addition, they showed that the radial velocity of this source is consistent with the SMC, which strongly supports that this source is located in the SMC. Because of the similarity between the Swift X-ray spectra and the SSS phase of novae, they concluded that this source is a binary system consisting of a WD and a Be star. We also analyzed the spectral energy distribution of the optical counterpart and found that it is consistent with that of a B-type star. The optical spectrum taken by SMARTS showed clear emission lines of $H_{\alpha}$ and $H_{\beta}$, confirming the conclusion of Li et al. (2012).

We fit the Swift XRT spectra with absorbed blackbody or Mekal models. Neither model was strongly preferred from the statistics. However, the evolution of the temperature of the Mekal model shows an unexplained decrease at three to seven days, while the temperature and radius of the blackbody fits can be understood as being caused by the shrinking photosphere. Here, the radius decreased from $\sim 10^{4} \mathrm{~km}$ to $\sim 100 \mathrm{~km}$, while the temperature increased from $\sim 0.06 \mathrm{keV}$ to $\sim 0.1 \mathrm{keV}$. Therefore, we conclude that the spectra were basically blackbody-like, and, hence, can be identified as a super-soft X-ray phase of a nova. This conclusion is the same as Li et al. (2012). Fits using WD atmosphere models (Rauch et al. 2010; van Rossum 2012) would allow further insights, although a trial with the Tübingen WD model failed to improve the fits, because of the computational upper limit for the temperature (Li et al. 2012). Most likely, more sophisticated spectral models like atmosphere models would improve the fits. Li et al. (2012) reported that adding a broad emission line at $\sim 0.7 \mathrm{keV}$ and an absorption edge at $0.89 \mathrm{keV}$ improves the fit for the spectrum at 1.54 days significantly, which also supports our interpretation that the early X-ray emission is due to the SSS phase of novae.

The SSS phase spectra from the first Swift XRT follow-up observation at 0.44 days and the simultaneous optical enhanced emission obtained by Swift UVOT are unusual in the evolution of standard novae. Nonetheless, if the optically enhanced emission is the photospheric emission of nova ejecta as in standard novae, the speed class indicator parameter of $t_{d, 2}$ means that it is the second fastest nova next to U Sco (1.2 days; Schwarz et al.
2011) and an equal record to V838 Her (two days; Schwarz et al. 2011). The peak absolute magnitude $(-5.04 \pm 0.07)$ at 0.44 days in the first Swift UVOT observation is four magnitudes fainter than those of typical novae $(-9.016 \pm 0.008)$, expected from the maximum magnitude rate of decline relation (della Valle \& Livio 1995). If this enhanced optical emission was photospheric emission from the nova ejecta, it suggests a low ejecta mass in this nova explosion. In the subsequent discussion below, we will show that the enhanced optical emission is not photospheric emission, however, the conclusion of the low ejecta mass remains correct.

\subsection{Shock Heating Mechanism}

Optically thin thermal X-ray emission of novae is usually explained by a shock-heating mechanism at a blast wave produced by the nova explosion. The recurrent nova, RS Ophiuchi, in 2006, exhibited the most luminous optically thin thermal X-ray emission $\left(1 \times 10^{36} \mathrm{erg} \mathrm{s}^{-1}\right)$ among novae that has ever been observed (Sokoloski et al. 2006). The luminosity of the X-ray outburst of MAXI J0158-744 was, surprisingly, four orders of magnitude larger than this. Li et al. (2012) explained the luminosity of MAXI J0158-744 by the shockheating mechanism, however, their explanation has difficulties. They tried to explain the observed luminosity of $\sim 10^{39} \mathrm{erg} \mathrm{s}^{-1}$ at the time of the first GSC scan (scan-ID M+0; Table 1) using Equation (1) of Li et al. (2012), where the radius of the shock wave $r_{s} \sim 1.5 \times 10^{13} \mathrm{~cm}$ is assumed. However, for the shock wave to expand to this radius within $\Delta t_{r}=t_{1}-t_{-1}=5.5 \times 10^{3} \mathrm{~s}$ (Table 1), the shock wave velocity must be exceptionally large ( $V_{s}=r_{s} / \Delta t_{r} \sim 3 \times 10^{4} \mathrm{~km} \mathrm{~s}^{-1}$ ) for novae. In addition, if the velocity of the shock wave was such a large value, the temperature of the plasma thermalized by the shock wave becomes very high $\left(k T=\left(3 m_{p} \mu V_{s}^{2} / 16\right) \sim 1 \mathrm{MeV}\right.$, where $m_{p}$ is the proton mass, and $\mu$ is the mean molecular weight ${ }^{25}$ ), which is contradictory to the observed soft spectrum of the outburst $(k T \sim 1 \mathrm{keV})$.

We further discuss the shock heating scenario, considering the very rapid rise time within $\Delta t_{r} \mathrm{~s}$ and the observed low temperature $(0.97 \mathrm{keV}$; Table 2$)$. We set the onset time of the nova explosion $t_{0}$ between the last scan time of the scanID $\mathbf{M}-1\left(t_{-1}\right.$; Table 1$)$ and the first scan time of the scanID $\mathrm{M}+0\left(t_{1}\right.$; Table 1$)$. The elapsed time from $t_{0}$ to $t_{1}$ is

\footnotetext{
$25 \mu=\left(A_{r 1} / f_{a 0}+f_{a 1}\right)=0.61$ for a typical $\mathrm{SMC}$ abundance ( $\mathrm{abund}=0.1$; Carrera et al. 2008). Here, $f_{a 0}=\sum_{i} a_{i}=1.10, f_{a 1}=\sum_{i} Z_{i} a_{i}=1.20$, and $A_{r 1}=\sum_{i} A_{r, i} a_{i}=1.40$ (Anders \& Grevesse 1989), where $Z_{i}, a_{i}$, and $A_{r, i}$ are the atomic number, abundance, and relative atomic mass of the $i$ th element. The abundance is defined by the ratio of the number densities of the $i$ th element and hydrogen $\left(a_{i}=n_{\mathrm{X}_{i}} / n_{\mathrm{H}}\right)$.
} 
$t_{\mathrm{e}, 1}=t_{1}-t_{0}<\Delta t_{r} \mathrm{~s}$. We check whether the observed emission measure $\left(\mathrm{EM} \equiv \int n_{e} n_{p} d V=1.0 \times 10^{63} \mathrm{EM}_{63} d_{60}{ }^{2} \mathrm{~cm}^{-3}\right.$, where $\mathrm{EM}_{63}=2.4$ for the Mekal model fit with free abund in Table 2 and $d_{60} \simeq 1$; here, $n_{e}$ and $n_{p}$ are the number densities of electrons and protons, respectively) can be produced at $t_{\mathrm{e}, 1}$ by considering two simple geometries, a filled sphere and spherical shell. Here, we assume the circumbinary space is filled with a fully ionized electron-ion plasma with a constant density. We assume the constant shock velocity $V_{s}$, and then the distance $\left(R_{1}\right)$ of the shock wave front reaching from the surface of the WD at the time $t_{\mathrm{e}, 1}$ is

$$
R_{1}=t_{\mathrm{e}, 1} \times V_{s}<\Delta t_{r} \times V_{s}=5.5 \times 10^{11} V_{s 3} \mathrm{~cm},
$$

where $V_{s}=10^{3} V_{s 3} \mathrm{~km} \mathrm{~s}^{-1}\left(V_{s 3} \simeq 1\right)$.

If the shape of the emission region is a filled sphere, the emission measure at $t_{\mathrm{e}, 1}$ is written as $\mathrm{EM}=(4 / 3) \pi R_{1}{ }^{3} n_{p}{ }^{\prime} n_{e}{ }^{\prime}=$ (4/3) $\pi R_{1}{ }^{3} f_{a 1} n_{p}{ }^{\prime 2}$ (hereafter, we add " "” for the physical value after the shock). Since we assume that the initial X-ray outburst is an optically-thin emission, the condition of the optical depth is $n_{e}{ }^{\prime} \sigma_{T} R_{1}=f_{a 1} n_{p}{ }^{\prime} \sigma_{T} R_{1}<1$, where $\sigma_{T}$ is the Thomson cross section. By removing $n_{p}{ }^{\prime}$, we obtain $R_{1}>$ $(3 \mathrm{EM} / 4 \pi) \sigma_{T}^{2} f_{a 1}=1.1 \times 10^{14} f_{a 1} \mathrm{EM}_{63} d_{60}{ }^{2} \mathrm{~cm}$. Therefore, it is impossible to produce the observed emission measure at $t_{1}$ in the usual shock velocity $\left(V_{s 3} \sim 1-10\right.$; Schwarz et al. 2011; Warner 2008), even at the speed of light.

Next, if the shape of the emission region is a spherical shell with a depth of $\delta R_{1}$, the emission measure at $t_{\mathrm{e}, 1}$ is written as $\mathrm{EM}=4 \pi R_{1}{ }^{2} \delta R_{1} n_{p}{ }^{\prime} n_{e}{ }^{\prime}$. The condition of the optical depth is $n_{e}{ }^{\prime} \sigma_{T} \delta R_{1}=f_{a 1} n_{p}{ }^{\prime} \sigma_{T} \delta R_{1}<1$. By removing $n_{p}{ }^{\prime}$, we obtain $R_{1}>(\mathrm{EM} / 4 \pi) \sigma_{T}^{2}\left(\delta R_{1} / R_{1}\right) f_{a 1}=$ $3.6 \times 10^{13} f_{a 1} \mathrm{EM}_{63} d_{60}{ }^{2}\left(\delta R_{1} / R_{1}\right) \mathrm{cm}$. Using condition 1 , the depth of the shell is limited to $\left(\delta R_{1} / R_{1}\right)<1.5 \times$ $10^{-2} d_{60}{ }^{-2} V_{s 3} \mathrm{EM}_{63}{ }^{-1} f_{a 1}^{-1}$. Therefore, the emission region is a thin shell. On the other hand, removing $\delta R_{1}$ and using condition 1 , we obtain

$$
\begin{aligned}
n_{p}{ }^{\prime}> & \frac{\mathrm{EM} \sigma_{T}}{4 \pi R_{1}^{2}}>\frac{\mathrm{EM} \sigma_{T}}{4 \pi\left(\Delta t_{r} V_{s}\right)^{2}} \\
& =1.8 \times 10^{14} d_{60}{ }^{2} V_{s 3}{ }^{-2} \mathrm{EM}_{63} \mathrm{~cm}^{-3} .
\end{aligned}
$$

In this high density, the shock velocity can be estimated simply from the observed temperature of $1.0 k T_{1.0} \mathrm{keV}$ $\left(k T_{1.0}=0.97\right.$; Table 2) using the shock condition by $V_{s}=$ $\left(16 k_{B} T_{d} / 3 m_{p} \mu\right)^{1 / 2}=7.1 \times 10^{2} k T_{1.0}{ }^{1 / 2} \mu^{-1 / 2} \mathrm{~km} \mathrm{~s}^{-1}$, where $T_{d}$ is the temperature of the shock-heated gas in the down-stream region.

The radiative cooling time scale by free-free process is

$$
\begin{aligned}
t_{\mathrm{cool}} & =3\left(\frac{3 m_{e}}{2 \pi}\right)^{1 / 2} \frac{3 h m_{e} c^{3}}{2^{5} \pi e^{6}}\left(k_{B} T_{e}\right)^{1 / 2} n_{p}^{\prime-1} \bar{g}_{B}^{-1} f_{b} \\
& =2.1 \times 10^{19}\left(k_{B} T_{e}\right)^{1 / 2} n_{p}^{\prime-1} f_{b},
\end{aligned}
$$

where $h, c$, and $\overline{g_{B}}$ are Planck constant, speed of light, and averaged Gaunt factor, respectively, and we set $\overline{g_{B}}=1.2$ (Rybicki $\&$ Lightman 1979). Here, $f_{b}=\left(f_{a 0}+f_{a 1} / 2 f_{a 1} f_{a 2}\right)\left(f_{a 2}=\right.$ $\left.\sum_{i} Z_{i}^{2} a_{i}\right)$ is 0.68 for a typical SMC abundance. From condition 2 , the $t_{\text {cool }}$ is limited to $t_{\text {cool }}<4.6 d_{60}{ }^{-2} V_{s 3}^{2} \mathrm{EM}_{63}{ }^{-1} k T_{1.0}{ }^{1 / 2} f_{b}$ s. Since the cooling time scale is short, the width of the emitting shell is simply written as $\delta R_{1}=(1 / 4) V_{s} \times t_{\text {cool }}$, where $(1 / 4) V_{s}$ is the velocity of the post-shock region in the rest frame of the shock wave. Using $\delta R_{1}=\left(\mathrm{EM} / 4 \pi R_{1}^{2} n_{p}^{\prime 2} f_{a 1}\right)$ and Equation (3), we derive the relation between $n_{p}^{\prime}$ and $R_{1}$, $n_{p}^{\prime}=3.8 \times 10^{39} \times R_{1}^{-2} d_{60}{ }^{2} V_{s 3}{ }^{-1} \mathrm{EM}_{63} k T_{1.0}{ }^{-1 / 2} f_{a 1}^{-1} f_{b}^{-1} \mathrm{~cm}^{-3}$. Using condition 1 , the density is limited to $n_{p}^{\prime}>1.3 \times$ $10^{16} d_{60}{ }^{2} V_{s 3}{ }^{-3} \mathrm{EM}_{63} k T_{1.0}{ }^{-1 / 2} f_{a 1}^{-1} f_{b}^{-1} \mathrm{~cm}^{-3}$. By setting $k T_{1.0}=$ $0.97, \mathrm{EM}_{63}=2.4, d_{60}=1$ (Table 2 ) and assuming a typical SMC abundance (abund $=0.1$ ), we obtain $n_{p}^{\prime}>5.2 \times$ $10^{16} \mathrm{~cm}^{-3}$. This density is much larger than that in the stellar wind and even that in a circumstellar equatorial disk around a Be star, typically $\lesssim 10^{13} \mathrm{~cm}^{-3}$ (Waters et al. 1988). Therefore, we conclude that the shock heating scenario cannot explain the soft X-ray outburst observed by MAXI.

\subsection{Thermonuclear Runaways at the Ignition Phase}

We thus instead propose another scenario to explain this outburst by invoking an extraordinary massive WD. A more massive WD has a smaller radius (Nauenberg 1972), and thus a higher surface gravity leading to a higher pressure in the accumulated mass. The nova explosion on a massive WD is triggered by less fuel, and results in a short nova duration. The observed SSS phase of MAXI J0158-744 started earlier $(<0.44$ days) and lasted a shorter time ( $\sim$ one month) than other fast novae (Hachisu \& Kato 2006; Schwarz et al. 2011; Li et al. 2012). The earliest turn-on of a SSS phase observed so far was 10 days in U Sco (Schwarz et al. 2011) and $11 \pm 5$ days in 1 of 60 novae in M31 (Henze et al. 2011). The extremely early SSS phase of MAXI J0158-744 is unexpected in models of novae on typical solar mass WDs (Hachisu \& Kato 2006). It suggests an unusually low ejecta mass in the nova explosion, and thus an unusually massive WD near the Chandrasekhar mass. It might even suggest a super-Chandrasekhar mass. According to theoretical models (Yoon \& Langer 2004; Hachisu et al. 2012), WDs can acquire super-Chandrasekhar masses up to 2.3-2.7 $M_{\odot}$, if they rotate differentially.

With this new perspective, we propose to interpret the initial super-Eddington X-ray outburst as an ignition phase of a nova just after the TNR, a fireball phase (Starrfield et al. 2008, 1998; Krautter 2008a, 2008b). In this process, the thermal energy produced by the TNR is conveyed by the convection and released outside the envelope with a timescale of $\sim 100 \mathrm{~s}$, characterized by the half-lives of unstable nuclei (Figure 5). In novae on a WD with a usual mass, transient soft X-ray emission $(<0.1 \mathrm{keV})$ for $\sim 100-1000 \mathrm{~s}$ just after the TNR is theoretically expected (Starrfield et al. 2008), but has not yet been observed. It is expected to reach about 10 times the Eddington luminosity (Starrfield et al. 2008). For a very massive WD, we speculate that the TNR would produce more luminous X-ray emission with higher temperature because of a smaller amount of the envelope at the ignition phase of a nova.

In this scenario, blackbody-like X-ray emission is expected at the ignition phase. In the spectral analysis (Table 2), we obtained the radius of the photosphere as $r_{\mathrm{ph}} \sim 10^{3} r_{\mathrm{ph}, 8} \mathrm{~km}$ $\left(r_{\mathrm{ph}, 8} \simeq 1\right)$. The rate of mass ejection $(\dot{M})$ can be estimated from this radius. From the continuity equation for the distribution of ejecta around the WD, $4 \pi r^{2} \rho V_{e}=\dot{M}$, where $\dot{M}$ is a rate of mass ejection from the WD and constant in the radial distance $(r)$, and $\rho=\sum A_{r, i} a_{i} m_{p} n_{p}=A_{r 1} m_{p} n_{p}$ is mass density, the number density of protons is written by

$$
n_{p}=\frac{\dot{M}}{4 \pi r^{2} A_{r 1} m_{p} V_{e}} .
$$




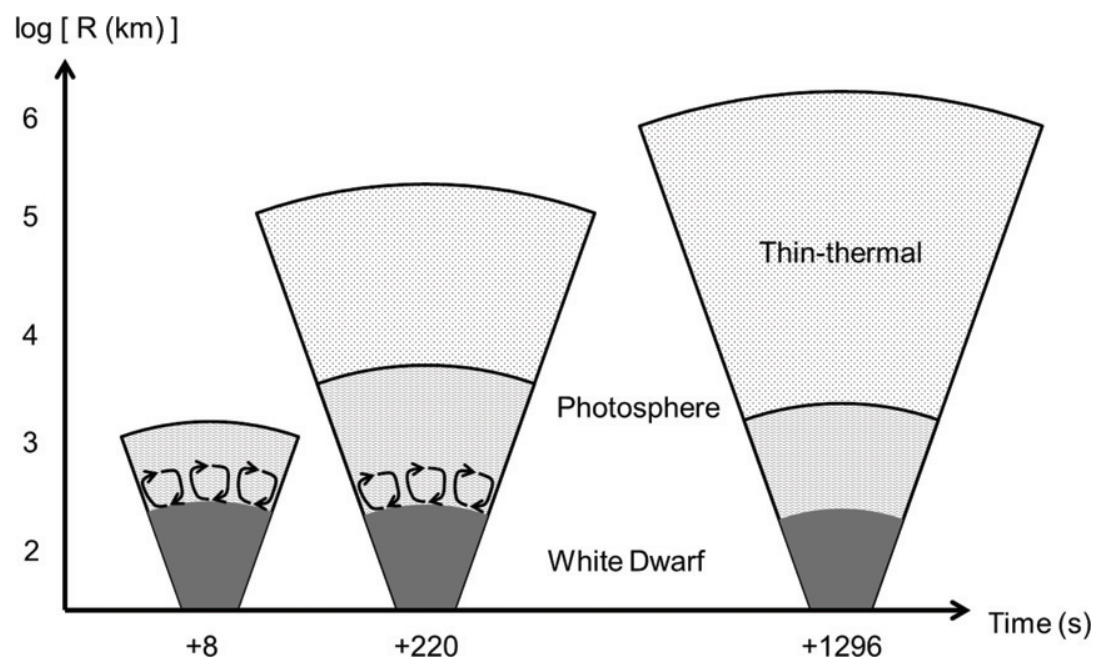

Figure 5. Schematic view of the ignition phase of the nova, MAXI J0158-744. The super-Eddington luminosity is produced by convection during the first $\sim 100 \mathrm{~s}$. The neon emission line at $+1296 \mathrm{~s}$ was produced from the optically thin thermal region filled with the ejecta dredged-up from the O-Ne WD.

The optical depth condition is written by

$$
\tau=\int_{r_{\mathrm{ph}}}^{\infty} n_{e} \sigma_{T} d r=\int_{r_{\mathrm{ph}}}^{\infty} f_{a 1} n_{p} \sigma_{T} d r=\frac{f_{a 1}}{A_{r 1}} \frac{\dot{M} \sigma_{T}}{4 \pi m_{p} V_{e} r_{\mathrm{ph}}}=1
$$

Here, $f_{a 1} / A_{r 1} \simeq Z_{10} / A_{r, 10} \simeq 0.5$ for large abund of neon. Therefore, $\dot{M}$ is obtained as

$$
\dot{M}=\frac{A_{r 1}}{f_{a 1}} \frac{4 \pi m_{p} V_{e} r_{\mathrm{ph}}}{\sigma_{T}} \simeq 6.4 \times 10^{17} V_{e 3} r_{\mathrm{ph}, 8} \mathrm{~g} \mathrm{~s}^{-1} .
$$

On the other hand, the reaction rate of mass producing nuclear energy $\left(\dot{M}_{f}\right)$ is related to the observed luminosity $\left(L_{X}\right)$ by $\eta \dot{M}_{f} c^{2}>L_{X}$, where $\eta=0.007$ and $L_{X}=10^{40} \mathrm{erg} \mathrm{s}^{-1}$ (Table 2). Then, $\dot{M}_{f}>L_{X} / \eta c^{2}=2 \times 10^{21} \mathrm{~g} \mathrm{~s}^{-1}$. Therefore, the relation $\dot{M}_{f} \gg \dot{M}$ is obtained, which means that energy produced by the TNR at the bottom of accreted layer can escape as X-ray photons efficiently with very small mass ejection, despite the super-Eddington luminosity.

Thus, there must be a some sort of mechanism to realize the super-Eddington luminosity with a small mass ejection. We infer a convection just after the TNR (Starrfield et al. 2008, 1998) for that mechanism, then we expect that future theoretical works of the TNR process, applied to the mass range near or over the Chandrasekhar limit, will clarify this mechanism. We also suspect that photon bubbles in highly magnetized atmospheres (Begelman 2001) may work to solve this problem. According to Begelman (2001), to produce the $\sim 100$ Eddington luminosity with small mass ejection, the magnetic pressure $P_{\text {mag }}$ must be $\sim 100$ times larger than the gas pressure $P_{\text {gas }}$. On the other hand, the gas pressure at the bottom of an accreted gas layer at an ignition of a nova is expected to be $P_{\text {gas }} \sim 10^{20}$ dyne $\mathrm{cm}^{-2}$ (Starrfield et al. 2008; Fujimoto 1982). Then the magnetic field $(B)$ necessary for the $\sim 100$ Eddington luminosity is $B=\left(8 \pi P_{\mathrm{mag}}\right)^{1 / 2} \sim\left(8 \pi 100 P_{\mathrm{gas}}\right)^{1 / 2} \sim 5 \times 10^{11} \mathrm{G}$. Such highly magnetized WDs with super-Chandrasekhar masses $\left(2.3-2.6 M_{\odot}\right)$ are predicted theoretically (Das \& Mukhopadhyay 2012).

Since the TNR process is expected to last for $\sim 100 \mathrm{~s}$ at the bottom of the accreted mass layer on the surface of WDs (Starrfield et al. 2008, 1998), the rate of mass ejection most likely peaked between the scans at $+220 \mathrm{~s}$ and $+1296 \mathrm{~s}$. It means that MAXI scans at $+8 \mathrm{~s}$ and $+220 \mathrm{~s}$ observed the photospheric expansion phase (B $\rightarrow$ C in Figure 1 of Kato \& Hachisu 1994), while the MAXI scan at $+1296 \mathrm{~s}$ observed the shrinking phase $(\mathrm{C} \rightarrow \mathrm{D}$ in the same figure). The strong neon emission line at +1296 s suggests that there was an optically thin thermal emission region surrounding the photosphere and filled with ejecta dredged-up from a massive O-Ne WD. Such ejecta may have been provided by the previous photospheric expansion. The existing models of the TNR do not predict this surrounding emission line region. This observation provides us new physical details.

In this scenario, the optical enhancement observed by Swift UVOT is no longer the usual photospheric emission of nova ejecta. Since the optical decay seems correlated with the decay of SSS X-ray emission (Figure 4, middle and bottom), it can be explained by the reprocessed emission from the X-ray irradiated circumstellar disk of the $\mathrm{Be}$ star. It is justified by the fact that the size of the optical enhanced emission $\left(6.6 \times 10^{11} \mathrm{~cm}\right.$; Section 3.4) is comparable to the disk scale height (Zorec et al. 2007).

\section{SUMMARY}

MAXI discovered an extraordinarily luminous soft X-ray transient, MAXI J0158-744, near the SMC on 2011 November 11. This source is a binary system consisting of a WD and a Be star at the distance of the SMC. MAXI detected it in three scans at $+8 \mathrm{~s},+220 \mathrm{~s}$, and $+1296 \mathrm{~s}$ after the trigger time. The $\mathrm{X}$-ray luminosity peaked on the second scan at $2 \times 10^{40} \mathrm{erg} \mathrm{s}^{-1}$ $(0.7-7.0 \mathrm{keV})$, which is two orders of magnitude brighter than the Eddington luminosity of a solar mass object. The spectrum of the third scan showed a He-like neon emission, suggesting that the emission contains an optically-thin thermal component and the WD is a massive O-Ne WD. While the X-ray outburst could be considered as a kind of nova on the basis of the luminosity and the spectral evolutions, the huge peak luminosity and the rapid rise time $\left(<5.5 \times 10^{3} \mathrm{~s}\right)$ are difficult to explain by shockinduced emission, accepted for optically-thin thermal emission in nova explosions observed so far. Instead, we propose the scenario that the X-ray outburst is the direct manifestation of the TNR process at the onset of the nova explosion, the socalled fireball phase. The super-Eddington X-ray outburst and the subsequent very early super-soft source phase indicate a 
small ejecta mass, implying the underlying WD is unusually massive near the Chandrasekhar limit, or possibly exceeding the limit.

We are grateful to the members of the MAXI and Swift operation teams. We thank K. Asano, I. Hachisu, D. N. Burrows, D. Takei, S. R. Kulkarni, Y. Maeda, and T. Shigeyama for discussions and comments. This research was partially supported by the Ministry of Education, Culture, Sports, Science and Technology (MEXT), Grant-in-Aid No. 23740147, 19047001, and Global-COE from MEXT "The Next Generation of Physics, Spun from Universality and Emergence" and "Nanoscience and Quantum Physics.” J.P.O., K.L.P. and N.P.M.K. acknowledge financial support from the UK Space Agency. J.A.K. acknowledges support from NASA. This work was supported by the Australian Research Council Discovery Projects funding scheme (project number DP120102393).

\section{REFERENCES}

Anders, E., \& Grevesse, N. 1989, GeCoA, 53, 197

Band, D., Matteson, J., Ford, L., et al. 1993, ApJ, 413, 281

Begelman, M. C. 2001, ApJ, 551, 897

Bohlin, R. C., Savage, B. D., \& Drake, J. F. 1978, ApJ, 224, 132

Burrows, D. N., Hill, J. E., Nousek, J. A., et al. 2005, SSRv, 120, 165

Carrera, R., Gallart, C., Aparicio, A., et al. 2008, AJ, 136, 1039

Cash, W. 1979, ApJ, 228, 939

Das, U., \& Mukhopadhyay, B. 2012, PhRvD, 86, 042001

della Valle, M., \& Livio, M. 1995, ApJ, 452, 704

Dickey, J. M., \& Lockman, F. J. 1990, ARA\&A, 28, 215

Fujimoto, M. Y. 1982, ApJ, 257, 752

Galloway, D. K., Muno, M. P., Hartman, J. M., Psaltis, D., \& Chakrabarty, D. 2008, ApJS, 179, 360

Gehrels, N., Chincarini, G., Giommi, P., et al. 2004, ApJ, 611, 1005

Hachisu, I., \& Kato, M. 2006, ApJS, 167, 59

Hachisu, I., Kato, M., Saio, H., \& Nomoto, K. 2012, ApJ, 744, 69

Henze, M., Pietsch, W., Haberl, F., et al. 2011, A\&A, 533, A52

Hilditch, R. W., Howarth, I. D., \& Harries, T. J. 2005, MNRAS, 357, 304

Hiroi, K., Ueda, Y., Isobe, N., et al. 2011, PASJ, 63, S677

Kalberla, P. M. W., Burton, W. B., Hartmann, D., et al. 2005, A\&A, 440,775

Kato, M., \& Hachisu, I. 1994, ApJ, 437, 802

Kennea, J. A., Hoversten, E. A., Curran, P., et al. 2011a, ATel, 3758

Kennea, J. A., Romano, P., Mangano, V., et al. 2011b, Proceedings of 4th International MAXI Workshop, The First Year of MAXI: Monitoring Variable X-ray Sources, arXiv:1101.6055

Kimura, M., Tomida, H., Sootome, T., et al. 2011, ATel, 3756

Kimura, M., Tsunemi, H., Tomida, H., et al. 2013, PASJ, 65, 14

Krautter, J. 2008a, in ASP Conf. Ser. 401, The Super-soft Phase in Novae in "RS Ophiuchi (2006) and the Recurrent Nova Phenomenon, ed. A. Evans, M. F. Bode, T. J. O’Brien, \& M. J. Darnley (San Francisco, CA: ASP), 139
Krautter, J. 2008b, in X-ray Emission from Classical Novae in Outburst in Classical Novae, ed. M. Bode \& A. Evans (2nd ed.; Cambridge: Cambridge Univ. Press), 232

Li, K. L., Kong, A. K. H., Charles, P. A., et al. 2012, ApJ, 761, 99

Matsuoka, M., Kawasaki, K., Ueno, S., et al. 2009, PASJ, 61, 999

Mewe, R., Gronenschild, E. H. B. M., \& van den Oord, G. H. J. 1985, A\&A, 62, 197

Mihara, T., Nakajima, M., Sugizaki, M., et al. 2011, PASJ, 63, S623

Morii, M., Kawai, N., Usui, R., et al. 2011a, PASJ, 63, S821

Morii, M., Kimura, M., Tomida, H., et al. 2011b, GCN Circ., 12555

Morrissey, P., Schiminovich, D., Barlow, T. A., et al. 2005, ApJL, 619, L7

Nakahira, S., Koyama, S., Ueda, Y., et al. 2012, PASJ, 64, 13

Nauenberg, M. 1972, ApJ, 175, 417

Negoro, H., Miyoshi, S., Ozawa, H., et al. 2010, in ASP Conf. Ser. 434, Astronomical Data Analysis Software and Systems XIX, ed. Y. Mizumoto, K. Morita, \& M. Ohishi (San Francisco, CA: ASP), 127

Nelson, T., Donato, D., Mukai, K., Sokoloski, J., \& Chomiuk, L. 2012, ApJL, 761, L16

Nishiyama, S., Haba, Y., Kato, D., et al. 2007, ApJ, 658, 358

Poole, T. S., Breeveld, A. A., Page, M. J., et al. 2008, MNRAS, 383, 627

Rauch, T., Orio, M., Gonzales-Riestra, R., et al. 2010, ApJ, 717, 363

Roming, P. W. A., Kennedy, T. E., Mason, K. O., et al. 2005, SSRv, 120,95

Rybicki, G. B., \& Lightman, A. P. 1979, Radiative Processes in Astrophysics (New York: Wiley)

Schlegel, D. J., Finkbeiner, D. P., \& Davis, M. 1998, ApJ, 500, 525

Schwarz, G. J., Ness, J.-U., Osborne, J. P., et al. 2011, ApJS, 197, 31

Sguera, V., Bazzano, A., Bird, A. J., et al. 2006, ApJ, 646, 452

Soderberg, A. M., Berger, E., Page, K. L., et al. 2008, Natur, 453, 469

Sokoloski, J. L., Luna, G. J. M., Mukai, K., \& Kenyon, S. J. 2006, Natur, 442,276

Starrfield, S., Iliadis, C., \& Hix, W. R. 2008, in Thermonuclear Processes in Classical Novae, ed. M. Bode \& A. Evans (2nd ed.; Cambridge: Cambridge Univ. Press), 77

Starrfield, S., Truran, J. W., Wiescher, M. C., \& Sparks, W. M. 1998, MNRAS, 296, 502

Sugizaki, M., Mihara, T., Serino, M., et al. 2011, PASJ, 63, S635

Tomida, H., Tsunemi, H., Kimura, M., et al. 2011, PASJ, 63, 397

Tsunemi, H., Tomida, H., Katayama, H., et al. 2010, PASJ, 62, 1371

van Rossum, D. R. 2012, ApJ, 756, 43

Walter, F. M., Battisti, A., Towers, S. E., Bond, H. E., \& Stringfellow, G. S. 2012, PASP, 124, 1057

Walter, F. M., Stringfellow, G. S., Sherry, W. H., \& Field-Pollatou, A. 2004, AJ, 128,1872

Warner, B. 1995, Novae in Eruption in Cataclysmic Variable Stars (Cambridge: Cambridge Univ. Press)

Warner, B. 2008, in Properties of Novae: An Overview in Classical Novae, ed. M. Bode \& A. Evans (2nd ed.; Cambridge: Cambridge Univ. Press), 16

Waters, L. B. F. M., van den Heuvel, E. P. J., Taylor, A. R., Habets, G. M. H. J., \& Persi, P. 1988, A\&A, 198, 200

Woods, P. M., \& Thompson, C. 2006, in Soft Gamma Repeaters and Anomalous X-ray Pulsars: Magnetar Candidates in Compact Stellar X-ray Sources, ed. W. Lewin \& M. van der Klis (Cambridge Astrophysics Series No. 39; Cambridge: Cambridge Univ. Press), 547

Yoon, S.-C., \& Langer, N. 2004, A\&A, 419, 623

Zorec, J., Arias, M. L., Cidale, L., \& Ringuelet, A. E. 2007, A\&A, 470, 239 